This item was submitted to Loughborough's Research Repository by the author.

Items in Figshare are protected by copyright, with all rights reserved, unless otherwise indicated.

\title{
Undular bore theory for the Gardner equation
}

PLEASE CITE THE PUBLISHED VERSION

http://dx.doi.org/10.1103/PhysRevE.86.036605

\section{PUBLISHER}

(C) American Physical Society

\section{VERSION}

VoR (Version of Record)

\section{PUBLISHER STATEMENT}

This work is made available according to the conditions of the Creative Commons Attribution-NonCommercialNoDerivatives 4.0 International (CC BY-NC-ND 4.0) licence. Full details of this licence are available at: https://creativecommons.org/licenses/by-nc-nd/4.0/

\section{LICENCE}

CC BY-NC-ND 4.0

\section{REPOSITORY RECORD}

Kamchatnov, A.M., Y.-H. Kuo, Tai-Chia Lin, T.-L. Horng, S.-C. Gou, Richard Clift, G.A. El, and Roger H.J. Grimshaw. 2019. "Undular Bore Theory for the Gardner Equation”. figshare. https://hdl.handle.net/2134/17005. 


\title{
Undular bore theory for the Gardner equation
}

\author{
A. M. Kamchatnov, ${ }^{1}$ Y.-H. Kuo, ${ }^{2}$ T.-C. Lin, ${ }^{2}$ T.-L. Horng, ${ }^{3}$ S.-C. Gou,${ }^{4}$ R. Clift, ${ }^{5}$ G. A. El,${ }^{5}$ and R. H. J. Grimshaw ${ }^{5}$ \\ ${ }^{1}$ Institute of Spectroscopy, Russian Academy of Sciences, Troitsk, Moscow Region, 142190 Russia \\ ${ }^{2}$ Department of Mathematics, National Taiwan University, Taipei, Taiwan \\ ${ }^{3}$ Department of Applied Mathematics, Feng Chia University, Taichung 40724, Taiwan \\ ${ }^{4}$ Department of Physics, National Changhua University of Education, Changhua 50058, Taiwan \\ ${ }^{5}$ Department of Mathematical Sciences, Loughborough University, Loughborough LE11 3TU, United Kingdom
}

(Received 15 May 2012; revised manuscript received 15 August 2012; published 18 September 2012)

\begin{abstract}
We develop modulation theory for undular bores (dispersive shock waves) in the framework of the Gardner, or extended Korteweg-de Vries (KdV), equation, which is a generic mathematical model for weakly nonlinear and weakly dispersive wave propagation, when effects of higher order nonlinearity become important. Using a reduced version of the finite-gap integration method we derive the Gardner-Whitham modulation system in a Riemann invariant form and show that it can be mapped onto the well-known modulation system for the Korteweg-de Vries equation. The transformation between the two counterpart modulation systems is, however, not invertible. As a result, the study of the resolution of an initial discontinuity for the Gardner equation reveals a rich phenomenology of solutions which, along with the KdV-type simple undular bores, include nonlinear trigonometric bores, solibores, rarefaction waves, and composite solutions representing various combinations of the above structures. We construct full parametric maps of such solutions for both signs of the cubic nonlinear term in the Gardner equation. Our classification is supported by numerical simulations.
\end{abstract}

DOI: 10.1103/PhysRevE.86.036605 PACS number(s): 46.90.+s, 47.35.Fg, 47.55.Hd, 92.10.Hm

\section{INTRODUCTION}

The Gardner equation

$$
u_{t}+6 u u_{x}-6 \alpha u^{2} u_{x}+u_{x x x}=0,
$$

is a fundamental mathematical model for the description of weakly nonlinear dispersive waves in situations when the higher order nonlinearity effects, described by the cubic term $-6 \alpha u^{2} u_{x}$, become important. It first arose as an auxiliary mathematical tool in the derivation of the infinite set of local conservation laws of the Korteweg-de Vries (KdV) equation [1] but has been shown later to describe nonlinear wave effects in a number of physical contexts including plasma physics [2,3], stratified fluid flows [4], and quantum fluid dynamics [5]. One of the most important and best known applications of the Gardner equation is the description of large-amplitude internal waves (see [4,6,7], and references therein). The coefficient $\alpha$ in (1) can be positive or negative depending on the physical problem under consideration. In the context of internal waves, this depends on the stratification (see [4]). In the particular case of a two-layer fluid it is always positive [8].

When $\alpha=0$ the Gardner equation (1) reduces to the KdV equation

$$
u_{t}+6 u u_{x}+u_{x x x}=0 .
$$

Using the change of variables

$$
w=u-\frac{1}{2 \alpha}, \quad x^{\prime}=x+\frac{3}{2 \alpha} t,
$$

one transforms (1) to the modified $\mathrm{KdV}(\mathrm{mKdV})$ equation

$$
w_{t}-6 \alpha w^{2} w_{x^{\prime}}+w_{x^{\prime} x^{\prime} x^{\prime}}=0
$$

(note, however, the change of boundary conditions at infinity). The Gardner equation (1) is invariant with respect to the transformation:

$$
u \rightarrow \frac{1}{\alpha}-u,
$$

which makes the existence of solutions of different polarity (e.g., "bright" and "dark" solitons) possible for the same system, depending on the initial conditions. This is markedly different from the properties of the $\mathrm{KdV}$ equation, which admits, for a given set of coefficients, solitary wave solutions of a fixed polarity, independently of the initial conditions.

The soliton solutions of the Gardner equation for both signs of $\alpha$ are well known and, along with the usual KdV-type bright and dark solitons, include tabletop solitons, breathers, algebraic solitons, and kinks (solibores). Much less is known about the dynamics of undular bores described by the Gardner equation. This problem is of significant theoretical and applied interest and is of particular importance in oceanography, where undular bores play a key role in the evolution of the internal tide (see [4,9]).

Undular bores are nonlinear expanding wave trains connecting two different basic flow states and exhibiting solitary waves near one of the edges. They are usually formed as a result of dispersive resolution of an initial discontinuity in fluid depth and/or velocity (see, e.g., $[10,11]$ ) or due to a resonant interaction of a fluid flow with localized topography (see, e.g., [12,13]). Formation of undular bores (also often called dispersive shock waves) is a generic physical phenomenon which has been observed not only in classical fluids but also in collisionless plasmas, Bose-Einstein condensates, and nonlinear optical media (see [14], and references therein).

The analytical description of undular bores is usually made in the framework of the Whitham modulation theory $[15,16]$ in which the asymptotic solution for the bore is sought in the form of a slowly varying periodic solution of the governing dispersive equation. The slow evolution of the modulation parameters (such as mean value, amplitude, wave number, etc.) 
is then governed by a hydrodynamic-type system of averaged equations, called the Whitham equations. The modulation description of the $\mathrm{KdV}$ undular bore was first constructed in the celebrated paper by Gurevich and Pitaevskii [17] and was later generalized to other dispersive systems both integrable (see, e.g., [18,19], and references therein) and nonintegrable [11,20-22].

The modulation system for the $\mathrm{KdV}$ equation can be represented in the Riemann invariant form $[15,16]$ which plays the key role in the Gurevich-Pitaevskii analytical construction of the $\mathrm{KdV}$ undular bore. For the $\mathrm{mKdV}$ equation (4) [both defocusing $(\alpha>0)$ and focusing $(\alpha<0)$ cases] the modulation system in Riemann invariants was derived in [23] using direct averaging of conservation laws and nontrivial algebraic manipulations leading to the diagonal structure. The spectral (finite-gap) approach to the derivation of the defocusing $\mathrm{mKdV}$ modulation system in the Riemann form was used in [24], where the modulation solution was obtained for the undular bore resolving the "cubic" wave breaking singularity. The undular bore theory for the focusing $(\alpha<0)$ mKdV equation (4) was constructed in [25]. It was shown in [25] that, along with the KdV-type cnoidal undular bores, in which the elliptic modulus $m$ varies together with the wave amplitude $a$ from $m=0, a=0$ at the trailing edge to $m=1$, $a=a^{+}$at the leading edge, $a^{+}>0$ being the amplitude of the lead solitary wave, the focusing mKdV equation supports another type of modulated solutions, termed trigonometric bores, in which $m=0$ throughout the whole wave train but the amplitude $a \neq 0$ and vanishes only at the trailing edge. It was also shown in [25] that the trigonometric bore is usually realized as part of a composite solution: either a combined cnoidal-trigonometric bore or a combination of a trigonometric bore and a simple rarefaction wave. Similar composite solutions were constructed in [26] for the complex $\mathrm{mKdV}$ equation [which is related to the defocusing nonlinear Schrödinger (NLS), rather than the KdV equation]. We stress that neither trigonometric bores nor composite modulation solutions exist in the KdV and NLS modulation theories. These new patterns owe their existence to the fact that the $\mathrm{mKdV}$ modulation system, unlike the KdV and NLS modulation systems, is neither strictly hyperbolic nor genuinely nonlinear [26]. Of course, the latter is not surprising if one remembers that the $\mathrm{KdV}$ and $\mathrm{mKdV}$ modulation systems are related by a noninvertible quadratic mapping [23], a modulation counterpart of the Miura transformation.

The Gardner equation, similar to the $\mathrm{KdV}$ and $\mathrm{mKdV}$ equations, is a completely integrable system, which implies that the Riemann invariants are, in principle, available for the associated modulation system. However, we are not aware of any publications containing a consistent and complete derivation of the Gardner modulation system in Riemann invariant form (we note that some particular results for the Riemann invariants via the mapping between the $\mathrm{KdV}$ and Gardner spectral problems can be found in [27]). Consequently, the full theory of the Gardner undular bores has not been constructed. Some analytical progress has only been made for the case when the coefficient $\alpha$ is sufficiently small so that the Gardner equation can be asymptotically reduced to the $\mathrm{KdV}$ equation via a near-identity transformation [28]. The undular bore solutions in this case are qualitatively similar to their $\mathrm{KdV}$ equation counterparts provided initial discontinuity is not very large. An interesting phenomenology of the Gardner undular bore solutions, beyond the $\mathrm{KdV}$ paradigm, was revealed in the numerical simulations in [29,30], where the problem of the transcritical flow of a stratified fluid was considered in the framework of the forced defocusing Gardner equation for a broad range of values for $\alpha<0$ and for the external forcing amplitude.

In this paper we derive the modulation system for the Gardner equation in the Riemann invariant form and construct a full classification of the asymptotic $(t \gg 1)$ solutions to the Gardner equation (1) with the initial conditions in the form of a step

$$
u(x, 0)= \begin{cases}u^{-}, & x<0, \\ u^{+}, & x>0 .\end{cases}
$$

We consider both signs of the coefficient $\alpha$ for the cubic nonlinear term.

In the $\mathrm{KdV}$ equation (2) theory, the resolution of the step (6) occurs via the generation of an undular bore if $u^{-}>u^{+}$or a rarefaction wave if $u^{-}<u^{+}$. For the Gardner equation we show that, due to the form of the nonlinear term in (1), the structure of the solutions to the initial value problem (1), (6) also depends on the positions of the initial step parameters $u^{+}, u^{-}$relative to the turning point $u=1 / 2 \alpha$ of the characteristic velocity $6 u(1-\alpha u)$ of the dispersionless limit of the Gardner equation. The full classification encompasses 16 possible cases (eight for each sign of $\alpha$ ). The wave patterns encountered include normal (bright) and reversed (dark) cnoidal undular bores, rarefaction waves, solibores (kinks), nonlinear trigonometric bores, and various combinations of the above patterns.

The structure of the paper is as follows. In Sec. II we undertake the derivation of two families of periodic solutions of the Gardner equation (1) corresponding to two signs of $\alpha$. The solutions are derived in the "natural" parametrization by considering the traveling wave ansatz $u=u(x-V t)$ in (1) and reducing it to an ordinary differential equation $u_{\xi}^{2}=Q(u), Q(u)$ being a polynomial of the fourth degree having (generally) four distinct roots $u_{1} \leqslant u_{2} \leqslant u_{3} \leqslant u_{4}$, only three of which are independent. The ordinary differential equation is integrated in terms of Jacobi elliptic functions and the harmonic $(m \rightarrow 0)$ and soliton $(m \rightarrow 1)$ limits for both families solutions are then investigated, $m$ being the modulus of the elliptic solution. Section III is devoted to the derivation of the Whitham modulation equations in Riemann invariant form. For that, we take advantage of the reduced version of the finite-gap integration method [18] to derive the "spectral" representation of the periodic solutions obtained in the previous section. The outcome is the set of relationships between the spectral parameters $r_{1}, r_{2}, r_{3}$ and the parameters $u_{1}, u_{2}, u_{3}, u_{4}$ characterizing the periodic solution [two possible sets of relationships $\mathbf{u}(\mathbf{r})$ are derived for each sign of $\alpha-$ this is a consequence of the invariance of the Gardner equation with respect to the transformation (5)]. The Whitham modulation equations are then derived for which $r_{j}$ 's are the Riemann invariants. In Sec. IV, based on the results obtained in Secs. II and III, we construct the full classifications of the solutions to the evolution of an initial discontinuity problem for the Gardner equation with $\alpha>0$ and $\alpha<0$. In Sec. $\mathrm{V}$ 
we draw conclusions from our analysis and outline possible applications of the obtained solutions.

\section{PERIODIC SOLUTION OF THE GARDNER EQUATION}

We start with a direct derivation of the periodic traveling wave solution of the Gardner equation (1). General expressions for such solutions can be found in [31]. Here we need a more detailed description suitable for our subsequent development of the undular bore theory. Introducing the substitution

$$
u=u(\xi), \quad \xi=x-V t,
$$

and integrating twice we arrive at a nonlinear oscillator equation

$$
u_{\xi}^{2}=\alpha u^{4}-2 u^{3}-V u^{2}+A u+B \equiv Q(u),
$$

where $A$ and $B$ are the integration constants. We shall sometimes refer to the polynomial $Q(u)$ in the right-hand side of (8) as a "potential curve" for the nonlinear oscillator described by (8). Let $Q(u)$ have four real roots

$$
u_{1} \leqslant u_{2} \leqslant u_{3} \leqslant u_{4}
$$

[the case when $Q(u)$ has two real and two complex conjugate roots corresponds to modulationally unstable solutions [23], so we do not consider it here]. The roots (9) are obviously related by the condition

$$
\sum_{i=1}^{4} u_{i}=\frac{2}{\alpha}
$$

and hence only three of them are independent. It is still convenient to keep all four $u_{j}$ 's in the subsequent formulas to preserve the symmetry of the expressions.

We should distinguish between two qualitatively different cases.

(a) Let $\alpha>0$. Then the periodic solution corresponds to the oscillations in the interval

$$
u_{2} \leqslant u \leqslant u_{3},
$$

where the polynomial $Q(u)$ is positive and

$$
\sqrt{\alpha}\left(\xi-\xi_{0}\right)=\int_{u}^{u_{3}} \frac{d u}{\sqrt{\left(u-u_{1}\right)\left(u-u_{2}\right)\left(u_{3}-u\right)\left(u_{4}-u\right)}} .
$$

$\xi_{0}$ being the integration constant (the initial phase). The possible configurations of the "potential" curve $Q(u)$ corresponding to qualitatively different traveling wave solutions are shown in Fig. 1.

The integral in (12) can be expressed in terms of the incomplete elliptic integral of the first kind and its inversion yields, after some algebra, the solution in terms of Jacobi elliptic functions:

$$
u=u_{2}+\frac{\left(u_{3}-u_{2}\right) \operatorname{cn}^{2}\left(\theta, m_{1}\right)}{1-\frac{u_{3}-u_{2}}{u_{4}-u_{2}} \operatorname{sn}^{2}\left(\theta, m_{1}\right)},
$$

where $\left(\xi_{0}=0\right)$

$$
\begin{aligned}
\theta & =\sqrt{\alpha\left(u_{3}-u_{1}\right)\left(u_{4}-u_{2}\right)}(x-V t) / 2, \\
m_{1} & =\frac{\left(u_{3}-u_{2}\right)\left(u_{4}-u_{1}\right)}{\left(u_{4}-u_{2}\right)\left(u_{3}-u_{1}\right)},
\end{aligned}
$$
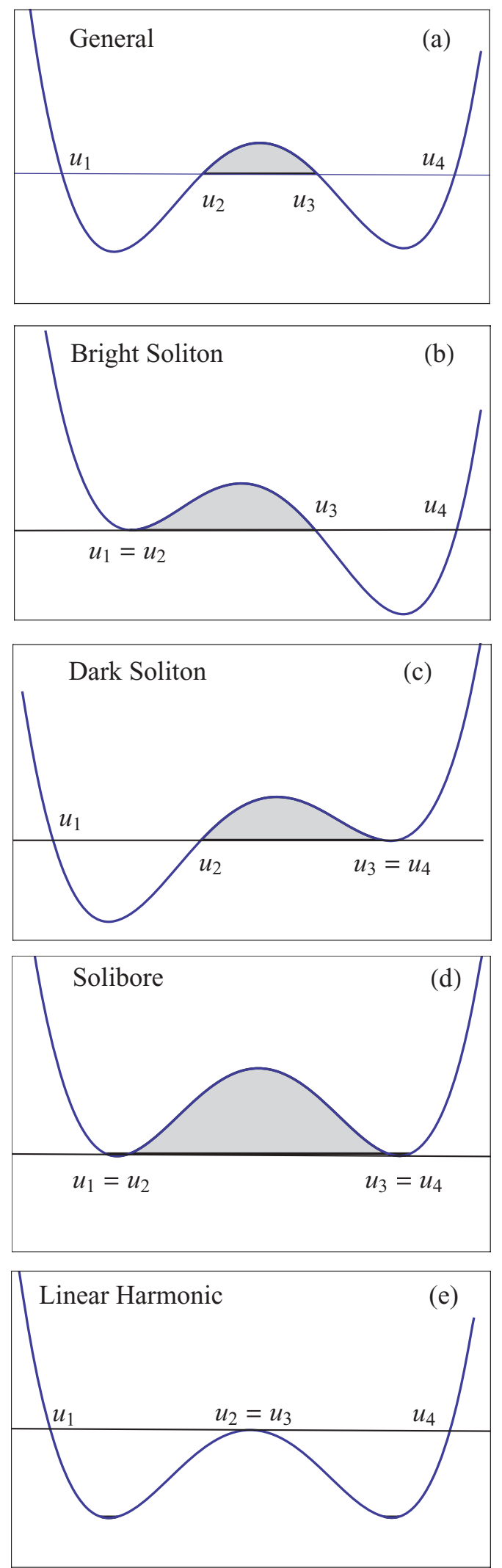

FIG. 1. (Color online) Potential curve $Q(u)$ configurations for the traveling wave solutions of the Gardner equation with $\alpha>0$. The oscillations occur between $u_{2}$ and $u_{3}$. (a) Periodic (elliptic) solution, (b) bright soliton, (c) dark soliton, (d) solibore, and (e) linear wave. 
and $V$ is given by

$$
V=\alpha\left(u_{1} u_{2}+u_{1} u_{3}+u_{1} u_{4}+u_{2} u_{3}+u_{2} u_{4}+u_{3} u_{4}\right) .
$$

The wavelength is given by the formula

$$
L=\frac{4 K\left(m_{1}\right)}{\sqrt{\alpha\left(u_{3}-u_{1}\right)\left(u_{4}-u_{2}\right)}},
$$

where $K\left(m_{1}\right)$ is the complete elliptic integral of the first kind. The soliton limit $m_{1} \rightarrow 1$ can be achieved in one of two ways: when $u_{1} \rightarrow u_{2}$ or when $u_{3} \rightarrow u_{4}$.

When $u_{2} \rightarrow u_{1}$ we obtain the bright soliton of elevation propagating against a constant background $u=u_{2}$ [see Fig. 1(b)],

$$
u(\xi)=u_{1}+\frac{u_{3}-u_{1}}{\cosh ^{2} \theta-\frac{u_{3}-u_{1}}{u_{4}-u_{1}} \sinh ^{2} \theta} .
$$

If, further, one has $u_{4}-u_{3} \ll u_{3}-u_{2}$, then the soliton (18) becomes a wide, "tabletop" soliton.

Analogously, for $u_{3} \rightarrow u_{4}$ we choose $\xi_{0}$ so that $u=u_{2}$ at $\xi=0$ and obtain

$$
u(\xi)=u_{4}-\frac{u_{4}-u_{2}}{\cosh ^{2} \theta-\frac{u_{4}-u_{2}}{u_{4}-u_{1}} \sinh ^{2} \theta} .
$$

This is a dark soliton on the constant background $u=u_{4}$ [see Fig. 1(c)]. If $u_{2}-u_{1} \ll u_{3}-u_{2}$ it assumes the form of a depression counterpart of the tabletop soliton.

If both $u_{2} \rightarrow u_{1}$ and $u_{3} \rightarrow u_{4}$ then the polynomial $Q(u)$ in the right-hand side of (8) has two double roots [see Fig. 1(d)], which implies that the solution assumes the form of a kink (a "solibore"). To study this limit, it is convenient to choose $\xi_{0}$ in such a way that $u=\left(u_{1}+u_{4}\right) / 2$ at $\xi=0$. As a result, an elementary integration of (8) yields two possible solutions:

$$
u(\xi)=u_{4}-\frac{u_{4}-u_{1}}{\exp \left[ \pm \sqrt{\alpha}\left(u_{4}-u_{1}\right) \xi\right]+1} .
$$

The lower sign corresponds to the kink with $u \rightarrow u_{4}$ as $\xi \rightarrow-\infty$ and $u \rightarrow u_{1}$ at $\xi \rightarrow \infty$; the upper sign yields the "antikink" with $u \rightarrow u_{1}$ as $\xi \rightarrow-\infty$ and $u \rightarrow u_{4}$ at $\xi \rightarrow \infty$. As follows from (10), the limiting constant states $u_{1}$ and $u_{4}$ are related by the condition $u_{1}+u_{4}=1 / \alpha$. The speed of the kink (solibore) propagation in both cases is $c=$ $\alpha^{-1}+2 \alpha u_{1} u_{4}$, which agrees with the shock speed obtained from the first conservation law $u_{t}+\left(3 u^{2}-2 \alpha u^{3}\right)_{x}=0$ of the dispersionless limit of the Gardner equation.

When $u_{3} \rightarrow u_{2}\left(m_{1} \rightarrow 0\right)$ [see Fig. 1(e)] the cnoidal wave (13) asymptotically transforms into a linear harmonic wave

$$
\begin{aligned}
u & \cong u_{2}+\frac{1}{2}\left(u_{3}-u_{2}\right) \cos [k(x-V t)], \\
k & =\sqrt{\alpha\left(u_{2}-u_{1}\right)\left(u_{4}-u_{2}\right)}, \\
V & =4 u_{2}+\alpha\left(u_{1} u_{4}-3 u_{2}^{2}\right) .
\end{aligned}
$$

(b) Let now $\alpha<0$. Then periodic solution corresponds to the oscillations in one of the two intervals,

$$
u_{1} \leqslant u \leqslant u_{2} \quad \text { or } \quad u_{3} \leqslant u \leqslant u_{4},
$$

where the polynomial $Q(u)$ assumes positive values. The possible configurations of the potential curve $Q(u)$ are shown in Fig. 2.
First we consider the case

$$
u_{1} \leqslant u \leqslant u_{2}
$$

so that

$$
\sqrt{|\alpha|}\left(\xi-\xi_{0}\right)=\int_{u_{1}}^{u} \frac{d u}{\sqrt{\left(u-u_{1}\right)\left(u_{2}-u\right)\left(u_{3}-u\right)\left(u_{4}-u\right)}} .
$$

A standard calculation yields

$$
u=u_{2}-\frac{\left(u_{2}-u_{1}\right) \mathrm{cn}^{2}\left(\theta, m_{2}\right)}{1+\frac{u_{2}-u_{1}}{u_{4}-u_{2}} \operatorname{sn}^{2}\left(\theta, m_{2}\right)},
$$

where

$$
\begin{aligned}
\theta & =\sqrt{|\alpha|\left(u_{3}-u_{1}\right)\left(u_{4}-u_{2}\right)}(x-V t) / 2, \\
m_{2} & =\frac{\left(u_{4}-u_{3}\right)\left(u_{2}-u_{1}\right)}{\left(u_{4}-u_{2}\right)\left(u_{3}-u_{1}\right)} .
\end{aligned}
$$

Now the wavelength is given by

$$
L=\frac{4 K\left(m_{2}\right)}{\sqrt{|\alpha|\left(u_{3}-u_{1}\right)\left(u_{4}-u_{2}\right)}} .
$$

In the soliton limit $u_{3} \rightarrow u_{2}\left(m_{2} \rightarrow 1\right)$ we get

$$
\begin{aligned}
& u=u_{2}-\frac{u_{2}-u_{1}}{\cosh ^{2} \theta+\frac{u_{2}-u_{1}}{u_{4}-u_{2}} \sinh ^{2} \theta}, \\
& V=2 u_{2}+\alpha\left(u_{1} u_{4}-3 u_{2}^{2}\right) .
\end{aligned}
$$

This is a dark, depression soliton.

The limit $m_{2} \rightarrow 0$ can be reached in two ways.

(1) If $u_{2} \rightarrow u_{1}$ [see Fig. 2(d)] we get asymptotically

$$
\begin{aligned}
u & \cong u_{2}-\frac{1}{2}\left(u_{2}-u_{1}\right) \cos [k(x-V t)], \\
k & =\sqrt{|\alpha|\left(u_{3}-u_{1}\right)\left(u_{4}-u_{1}\right)} \\
V & =4 u_{1}+\alpha\left(u_{3} u_{4}-3 u_{1}^{2}\right) .
\end{aligned}
$$

This is a small-amplitude harmonic limit.

(2) If $u_{4}=u_{3}$, but $u_{1} \neq u_{2}$ [see Fig. 2(e)] then we arrive at the nonlinear trigonometric solution

$$
u=u_{2}-\frac{\left(u_{2}-u_{1}\right) \cos ^{2} \theta}{1+\frac{u_{2}-u_{1}}{u_{3}-u_{2}} \sin ^{2} \theta},
$$

where

$$
\begin{aligned}
\theta & =\sqrt{|\alpha|\left(u_{3}-u_{1}\right)\left(u_{3}-u_{2}\right)}(x-V t) / 2, \\
V & =4 u_{3}+\alpha\left(u_{1} u_{2}-3 u_{3}^{2}\right) .
\end{aligned}
$$

If we take the limit $u_{2}-u_{1} \ll u_{3}-u_{1}$ in this solution, then we return to the particular case of the small-amplitude limit (30) with $u_{4}=u_{3}$, so that $k=\sqrt{|\alpha|}\left(u_{3}-u_{1}\right)$ and $V=4 u_{3}+\alpha\left(u_{1}^{2}-3 u_{3}^{2}\right) \equiv 4 u_{1}+\alpha\left(u_{3}^{2}-3 u_{1}^{2}\right)$. On the other hand, if we take here the limit $u_{2} \rightarrow u_{3}=u_{4}$, then the argument of trigonometric functions becomes small and we can approximate them by the first terms of their series expansions to get the dark algebraic soliton

$$
\begin{aligned}
& u=u_{2}-\frac{u_{2}-u_{1}}{1+|\alpha|\left(u_{2}-u_{1}\right)^{2}(x-V t)^{2} / 4}, \\
& V=2 u_{2}\left(1+\alpha u_{1}\right) .
\end{aligned}
$$

Now we consider the case

$$
u_{3} \leqslant u \leqslant u_{4},
$$



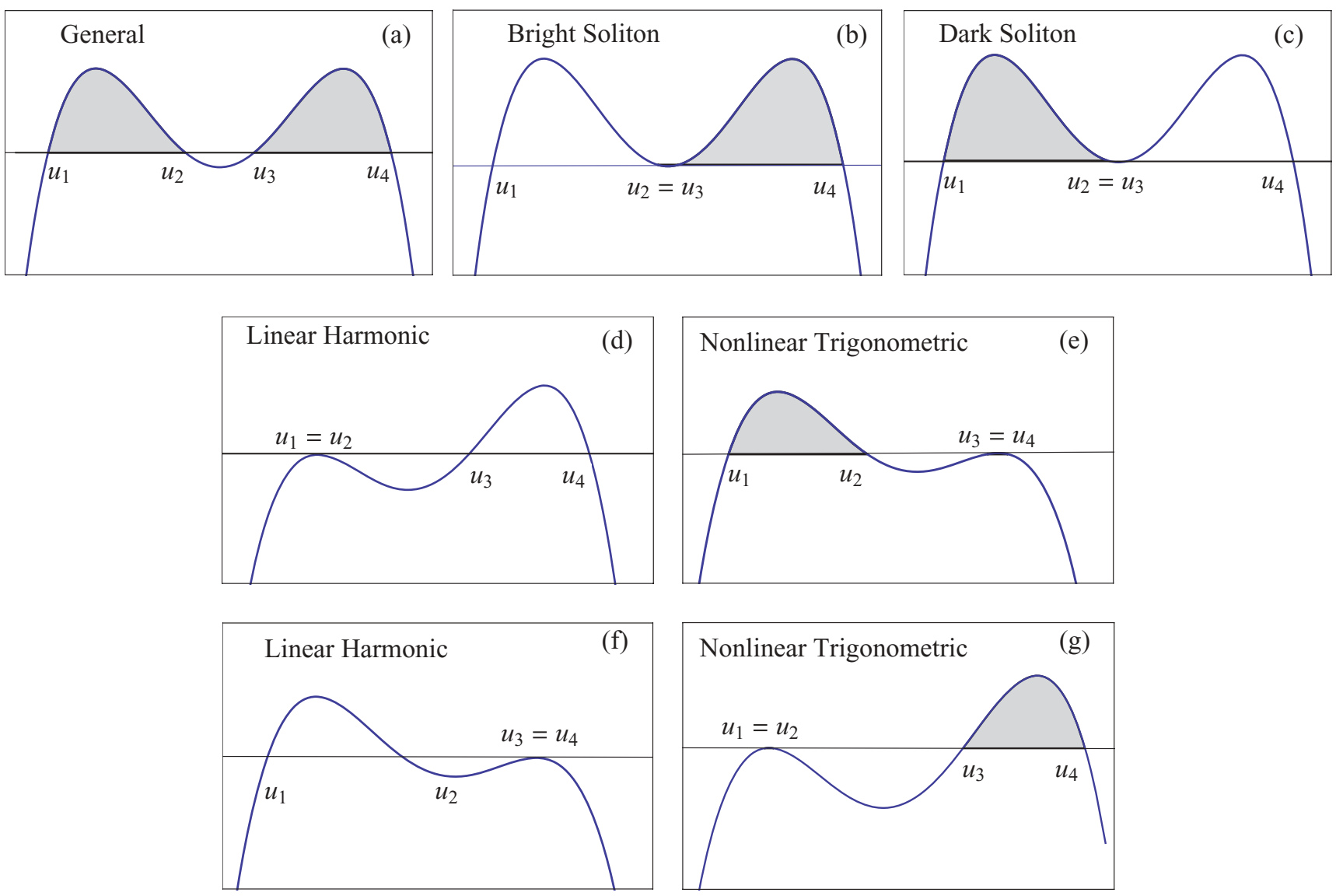

FIG. 2. (Color online) Potential curve $Q(u)$ configurations for the traveling wave solutions of the Gardner equation with $\alpha<0$ : (a) periodic (elliptic) solutions: $u_{1} \leqslant u \leqslant u_{2}$ or $u_{3} \leqslant u \leqslant u_{4}$; (b) bright soliton; (c) dark soliton; (d) linear harmonic wave, $m_{2}=0, a=0$ propagating about the background $u=u_{1}=u_{2}$; (e) nonlinear trigonometric wave, $m_{2}=0, a \neq 0, u_{1} \leqslant u \leqslant u_{2}$; (f) linear harmonic wave, $m_{2}=0, a=0$ propagating about $u=u_{3}=u_{4}$; and (g) nonlinear trigonometric wave, $m_{2}=0, a \neq 0, u_{3} \leqslant u \leqslant u_{4}$.

so that

$$
\sqrt{|\alpha|}\left(\xi-\xi_{0}\right)=\int_{u}^{u_{4}} \frac{d u}{\sqrt{\left(u-u_{1}\right)\left(u-u_{2}\right)\left(u-u_{3}\right)\left(u_{4}-u\right)}} .
$$

Again, the standard calculation yields

$$
u=u_{3}+\frac{\left(u_{4}-u_{3}\right) \operatorname{cn}^{2}\left(\theta, m_{2}\right)}{1+\frac{u_{4}-u_{3}}{u_{3}-u_{1}} \operatorname{sn}^{2}\left(\theta, m_{2}\right)} .
$$

In the soliton limit $u_{3} \rightarrow u_{2}(m \rightarrow 1)$ we get

$$
u=u_{2}+\frac{u_{4}-u_{2}}{\cosh ^{2} \theta+\frac{u_{4}-u_{2}}{u_{2}-u_{1}} \sinh ^{2} \theta}
$$

where

$$
\begin{aligned}
\theta & =\sqrt{|\alpha|\left(u_{2}-u_{1}\right)\left(u_{4}-u_{2}\right)}(x-V t) / 2, \\
V & =4 u_{2}+\alpha\left(u_{1} u_{4}-3 u_{2}^{2}\right) .
\end{aligned}
$$

This is a bright elevation soliton.

Again, there are two ways for getting the limit $m_{2} \rightarrow 0$.

(1) If $u_{4} \rightarrow u_{3}$ [see Fig. 2(f)], then we obtain a smallamplitude harmonic wave

$$
u=u_{3}+\frac{1}{2}\left(u_{4}-u_{3}\right) \cos [k(x-V t)],
$$

where

$$
\begin{aligned}
k & =\sqrt{|\alpha|\left(u_{3}-u_{1}\right)\left(u_{3}-u_{2}\right)} / 2, \\
V & =4 u_{3}+\alpha\left(u_{1} u_{2}-3 u_{3}^{2}\right) .
\end{aligned}
$$

(2) If $u_{2} \rightarrow u_{1}$ [see Fig. 2(g)], then we get another nonlinear trigonometric solution

$$
u=u_{3}+\frac{\left(u_{4}-u_{3}\right) \cos ^{2} \theta}{1+\frac{u_{4}-u_{3}}{u_{3}-u_{1}} \sin ^{2} \theta}
$$

where

$$
\begin{aligned}
\theta & =\sqrt{|\alpha|\left(u_{3}-u_{1}\right)\left(u_{4}-u_{1}\right)}(x-V t) / 2, \\
V & =4 u_{1}+\alpha\left(u_{3} u_{4}-3 u_{1}^{2}\right) .
\end{aligned}
$$

If we assume here $u_{4}-u_{3} \ll u_{4}-u_{1}$, then we reproduce the small-amplitude asymptotics (39) with $u_{2} \cong u_{1}$, so that $k=$ $\sqrt{|\alpha|}\left(u_{3}-u_{1}\right), V=4 u_{3}+\alpha\left(u_{1}^{2}-3 u_{3}^{2}\right)$. On the other hand, Eq. (41) in the limit $u_{3} \rightarrow u_{2}=u_{1}$ reduces to the algebraic bright soliton solution

$$
\begin{aligned}
u & =u_{1}+\frac{u_{4}-u_{1}}{1+|\alpha|\left(u_{4}-u_{1}\right)^{2}(x-V t)^{2} / 4}, \\
V & =2 u_{1}\left(1+\alpha u_{4}\right) .
\end{aligned}
$$

This completes the classification of stable periodic solutions and their limiting cases of the Gardner equation. 


\section{SPECTRAL PARAMETRIZATION OF THE PERIODIC SOLUTION}

\section{A. Motivation}

The periodic solution derived in the previous section is parametrized by four "integrals of motion" $u_{1} \leqslant u_{2} \leqslant u_{3} \leqslant$ $u_{4}$, which are related by the condition (10). In a strictly periodic solution these parameters $u_{j}$ are constants, but in a modulated wave, which we are interested in, they become slow functions of space coordinate $x$ and time $t$. Their evolution is then governed by the Whitham modulation equations (see $[15,16,18]$ ) which can be obtained by averaging the conservation laws of the Gardner equation over the periodic solution family (8), and which, generally speaking, have the form of a quasilinear (hydrodynamic type) system

$$
\frac{\partial u_{i}}{\partial t}+\sum_{j} v_{i j} \frac{\partial u_{j}}{\partial x}=0, \quad i, j=\text { any two of }\{1,2,3,4\} \text {. }
$$

Here the matrix elements $v_{i j}$ are functions of $\mathbf{u}=$ $\left(u_{1}, u_{2}, u_{3}, u_{4}\right)$ [note that one of the variables $u_{i}$ can be eliminated with the help of Eq. (10) but then the symmetry of the above expressions for the periodic solution will be lost]. The modulation system in the form (44) would be, however, completely impractical due to the highly complicated structure of the matrix elements $v_{i j}$-this is already the case even for the $\mathrm{KdV}$ equation (see $[15,16,18]$ ). Fortunately, for the Gardner equation this system can be transformed to the Riemann diagonal form

$$
\frac{\partial r_{k}}{\partial t}+v_{k}(\mathbf{r}) \frac{\partial r_{k}}{\partial x}=0, \quad k=1,2,3,
$$

where $r_{k}, k=1,2,3$, are the Riemann invariants. This is possible due to the fact that the Gardner equation is a completely integrable equation. Moreover, one can expect that, at least for $\alpha>0$, the Whitham system for the Gardner equation will be closely related (or even equivalent) to the Whitham system for the $\mathrm{KdV}$ equation. Indeed, for $\alpha>0$ the Gardner equation could be reduced, by a simple change of variables (3), to the defocusing $\mathrm{mKdV}$ equation, which, in its turn, is connected with the $\mathrm{KdV}$ equation by the Miura transform. As a result, for $\alpha>0$ the mKdV-Whitham system in the Riemann form is equivalent to that of the $\mathrm{KdV}$ equation (the result first obtained in [23]) and the same is true for the Whitham-Gardner system [27]. The Whitham equations (45) can be readily solved analytically, providing the necessary modulation solutions. The problem, however, is that one still needs to know the dependence of $u_{i}$ 's on the Riemann invariants $r_{1}, r_{2}, r_{3}$ for the Gardner equation to be able to find the modulations of the periodic traveling wave solutions obtained in the "natural" $u_{j}$ parametrization. This dependence for a particular case of the traveling wave solution (13) was found in [27] but the description in [27], being merely an illustration of a more general theory, is too brief and somewhat incomplete for our purposes, so below we present a detailed calculation, which also will not be restricted to the case $\alpha>0$.

The transformation $u_{i}=u_{i}(\mathbf{r})$ is most conveniently found using the spectral theory of the Gardner equation (1). The method of obtaining periodic (generally, quasiperiodic) solutions via the linear spectral problem associated with an integrable nonlinear dispersive equation is usually referred to as the finite-gap integration method. It is based on the highly nontrivial properties of quasiperiodic solutions of soliton equations which have only a finite number of bands (gaps) in their spectrum when considered as potentials in the associated spectral problem (see, e.g., [32]). In the context of the Whitham modulation theory the advantage of the finite-gap integration over the direct procedure of finding periodic solutions is that, if the end points of the spectral bands of the potential (quasiperiodic solution) are allowed to slowly vary with $x$ and $t$, they become the Riemann invariants of the modulation equations. The full finite-gap integration theory and the associated modulation theory, however, are quite technical and involve rather complicated algebraic-geometrical constructions on hyperelliptic Riemann surfaces (see, e.g., seminal paper [33] where this theory was developed for the first time for the $\mathrm{KdV}$ equation). However, in the single-phase periodic case of our interest a more simple, reduced version of the finite-gap integration method is available [18] enabling one to derive the required Riemann invariant parametrization for periodic solutions of integrable equations associated with $(2 \times 2)$ linear spectral problems.

\section{B. Spectral theory}

The finite-gap integration method is based on the possibility to represent the Gardner equation (1) as a compatibility condition of two linear systems (see, e.g., [18,32])

$$
\Psi_{x}=\mathbb{U} \Psi, \quad \Psi_{t}=\mathbb{V} \Psi,
$$

where

$$
\begin{aligned}
\Psi & =\left(\begin{array}{l}
\psi_{1} \\
\psi_{2}
\end{array}\right), \quad \mathbb{U}=\left(\begin{array}{cc}
F & G \\
H & -F
\end{array}\right), \quad \mathbb{V}=\left(\begin{array}{cc}
A & B \\
C & -A
\end{array}\right), \\
F & =\lambda, \quad G=-(1-\alpha u), \quad H=u, \\
A & =-4 \lambda^{3}-2 \lambda(1-\alpha u) u+u_{x}, \\
B & =4 \lambda^{2}(1-\alpha u)-2 \lambda \alpha u_{x}-\alpha u_{x x}+2(1-\alpha u)^{2} u, \\
C & =-4 \lambda^{2} u+2 \lambda u_{x}-u_{x x}-2(1-\alpha u) u^{2} .
\end{aligned}
$$

This means that the condition $\Psi_{x t}=\Psi_{t x}$ reduces to Eq. (1). (This mathematical property of the Gardner equation is similar to the properties of the $\mathrm{KdV}$ and $\mathrm{mKdV}$ equations; the relationship between Lax pairs for the $\mathrm{KdV}$ and $\mathrm{mKdV}$ hierarchies was discussed in [34].)

Calculations become somewhat simpler if we transform this matrix form of equations to their scalar counterparts (see [35])

$$
\psi_{x x}=\mathcal{A} \psi, \quad \psi_{t}=\mathcal{B} \psi,
$$

where

$$
\begin{aligned}
& \mathcal{A}=\left(\lambda+\frac{u_{x}}{2 u}\right)^{2}-(1-\alpha u) u-\left(\frac{u_{x}}{2 u}\right)_{x}, \\
& \mathcal{B}=-4 \lambda^{2}+2 \lambda \frac{u_{x}}{u}-\frac{u_{x x}}{u}-2(1-\alpha u) u .
\end{aligned}
$$

Then the second-order spectral equation in (50) has two basis solutions $\psi_{+}$and $\psi_{-}$and the "squared basis function"

$$
g=\psi_{+} \psi_{-}
$$


satisfies the third-order equation with a well-known integral

$$
\frac{1}{2} g g_{x x}-\frac{1}{4} g_{x}^{2}-\mathcal{A} g^{2}=P(\lambda) .
$$

In the finite-gap integration method the periodic solutions are distinguished by the condition that $P(\lambda)$ is a polynomial in $\lambda$. In our case we find that the one-phase periodic solution corresponds to the polynomial

$$
P(\lambda)=-\prod_{i=1}^{3}\left(\lambda^{2}-\lambda_{i}^{2}\right)=-\left(\lambda^{6}-s_{1} \lambda^{4}+s_{2} \lambda^{2}-s_{3}\right)
$$

and

$$
g=\lambda^{2}-g_{1} \lambda+g_{2},
$$

where the coefficients $g_{1}$ and $g_{2}$ are functions of $x$ and $t$. Substitution of Eqs. (55) and (56) into Eq. (54) with $\mathcal{A}$ given by Eq. (51) and equating of the coefficients of equal degrees of $\lambda$ at both sides of the resulting equation yields a set of equations for $g_{1}, g_{2}$, as well as for $u$ and its $x$ derivatives. Elimination of $g_{1}, g_{2}$, and of higher $x$ derivatives of $u$ from these equations gives after somewhat tedious calculations the equation

$$
\begin{aligned}
u_{x}^{2}= & \alpha u^{4}-2 u^{3}+2 s_{1} u^{2} \\
& +\left\{\frac{1}{\alpha^{2}}-\frac{2 s_{1}}{\alpha} \mp \sqrt{\left(1-4 \alpha \lambda_{1}^{2}\right)\left(1-4 \alpha \lambda_{2}^{2}\right)\left(1-4 \alpha \lambda_{3}^{2}\right)}\right\} u \\
& -\frac{1}{2 \alpha^{3}}\left[1-2 \alpha s_{1}-2 \alpha^{2}\left(s_{1}^{2}-4 s_{2}\right)\right. \\
& \left.\mp \sqrt{\left(1-4 \alpha \lambda_{1}^{2}\right)\left(1-4 \alpha \lambda_{2}^{2}\right)\left(1-4 \alpha \lambda_{3}^{2}\right)}\right] .
\end{aligned}
$$

The traveling periodic solution of Eq. (1) is then obtained by the replacement $x \mapsto x-V t$, where $V=-2 s_{1}$, that is,

$$
V=2\left(\lambda_{1}^{2}+\lambda_{2}^{2}+\lambda_{3}^{2}\right)=2\left(r_{1}+r_{2}+r_{3}\right),
$$

where we have introduced $r_{i}=\lambda_{i}^{2}, i=1,2,3$. The parameters $r_{j}$ are expected to become the Riemann invariants of the Whitham modulation equations.

We note that the representation (57) of the ordinary differential equation for a periodic solution, unlike that given by its equivalent (8), contains two possible signs. As a result, one needs to use different sets of relationships between $\left\{u_{i}\right\}$ and $\left\{r_{i}\right\}$ for different types of solutions, i.e., the mapping $\left\{u_{i}\right\} \mapsto$ $\left\{r_{i}\right\}$ is not one to one. To express the original parameters $u_{j}$ [the zeros of the polynomial in the right-hand side of equation (8)] in terms of the spectral parameters $r_{i}=\lambda_{i}^{2}$ we compare the two forms of the same ordinary differential equation defining the periodic solution, namely, Eqs. (8) and (57).

First let us consider the case $\alpha>0$. The example of the $\mathrm{mKdV}$ equation (see [24]) suggests the following expressions which can be verified by direct calculations:

$$
\begin{aligned}
& u_{1}=\frac{1}{2 \alpha}\left(1-\sqrt{1-4 \alpha r_{1}}-\sqrt{1-4 \alpha r_{2}}+\sqrt{1-4 \alpha r_{3}}\right), \\
& u_{2}=\frac{1}{2 \alpha}\left(1-\sqrt{1-4 \alpha r_{1}}+\sqrt{1-4 \alpha r_{2}}-\sqrt{1-4 \alpha r_{3}}\right), \\
& u_{3}=\frac{1}{2 \alpha}\left(1+\sqrt{1-4 \alpha r_{1}}-\sqrt{1-4 \alpha r_{2}}-\sqrt{1-4 \alpha r_{3}}\right), \\
& u_{4}=\frac{1}{2 \alpha}\left(1+\sqrt{1-4 \alpha r_{1}}+\sqrt{1-4 \alpha r_{2}}+\sqrt{1-4 \alpha r_{3}}\right),
\end{aligned}
$$

in the case of the upper sign in Eq. (57) and

$$
\begin{aligned}
& u_{1}=\frac{1}{2 \alpha}\left(1-\sqrt{1-4 \alpha r_{1}}-\sqrt{1-4 \alpha r_{2}}-\sqrt{1-4 \alpha r_{3}}\right), \\
& u_{2}=\frac{1}{2 \alpha}\left(1-\sqrt{1-4 \alpha r_{1}}+\sqrt{1-4 \alpha r_{2}}+\sqrt{1-4 \alpha r_{3}}\right), \\
& u_{3}=\frac{1}{2 \alpha}\left(1+\sqrt{1-4 \alpha r_{1}}-\sqrt{1-4 \alpha r_{2}}+\sqrt{1-4 \alpha r_{3}}\right), \\
& u_{4}=\frac{1}{2 \alpha}\left(1+\sqrt{1-4 \alpha r_{1}}+\sqrt{1-4 \alpha r_{2}}-\sqrt{1-4 \alpha r_{3}}\right),
\end{aligned}
$$

in the case of the lower sign. In both cases the zeros $u_{i}$ are ordered according to (9) provided

$$
\lambda_{1}^{2} \leqslant \lambda_{2}^{2} \leqslant \lambda_{3}^{2} \text { or, equivalently, } r_{1} \leqslant r_{2} \leqslant r_{3} .
$$

For both cases (59) and (60) the inverse formulas are simply

$$
\begin{aligned}
& r_{1}=\frac{\alpha}{4}\left(u_{1}+u_{2}\right)\left(u_{3}+u_{4}\right), \\
& r_{2}=\frac{\alpha}{4}\left(u_{1}+u_{3}\right)\left(u_{2}+u_{4}\right), \\
& r_{3}=\frac{\alpha}{4}\left(u_{2}+u_{3}\right)\left(u_{1}+u_{4}\right) .
\end{aligned}
$$

The existence of two sets (59), (60) of the traveling wave parameters corresponding to the same set of the spectral parameters $r_{j}$ is due to the invariance of the Gardner equation with respect to the transformation (5). Indeed, the set of relationships (60) can be obtained from (59) by applying the transformation $u_{j} \rightarrow 1 / \alpha-u_{j}$ and then reordering the resulting set.

In the case of $\alpha<0$ the expressions remain the same but their order corresponding to (9) is different. For the upper sign in (57) we obtain

$$
\begin{aligned}
& u_{1}=\frac{1}{2 \alpha}\left(1+\sqrt{1-4 \alpha r_{1}}+\sqrt{1-4 \alpha r_{2}}+\sqrt{1-4 \alpha r_{3}}\right), \\
& u_{2}=\frac{1}{2 \alpha}\left(1-\sqrt{1-4 \alpha r_{1}}-\sqrt{1-4 \alpha r_{2}}+\sqrt{1-4 \alpha r_{3}}\right), \\
& u_{3}=\frac{1}{2 \alpha}\left(1-\sqrt{1-4 \alpha r_{1}}+\sqrt{1-4 \alpha r_{2}}-\sqrt{1-4 \alpha r_{3}}\right), \\
& u_{4}=\frac{1}{2 \alpha}\left(1+\sqrt{1-4 \alpha r_{1}}-\sqrt{1-4 \alpha r_{2}}-\sqrt{1-4 \alpha r_{3}}\right),
\end{aligned}
$$

and for the lower sign

$$
\begin{aligned}
& u_{1}=\frac{1}{2 \alpha}\left(1-\sqrt{1-4 \alpha r_{1}}+\sqrt{1-4 \alpha r_{2}}+\sqrt{1-4 \alpha r_{3}}\right), \\
& u_{2}=\frac{1}{2 \alpha}\left(1+\sqrt{1-4 \alpha r_{1}}-\sqrt{1-4 \alpha r_{2}}+\sqrt{1-4 \alpha r_{3}}\right), \\
& u_{3}=\frac{1}{2 \alpha}\left(1+\sqrt{1-4 \alpha r_{1}}+\sqrt{1-4 \alpha r_{2}}-\sqrt{1-4 \alpha r_{3}}\right), \\
& u_{4}=\frac{1}{2 \alpha}\left(1-\sqrt{1-4 \alpha r_{1}}-\sqrt{1-4 \alpha r_{2}}-\sqrt{1-4 \alpha r_{3}}\right) .
\end{aligned}
$$

Now the inverse formulas are

$$
\begin{aligned}
& r_{1}=\frac{\alpha}{4}\left(u_{1}+u_{4}\right)\left(u_{2}+u_{3}\right), \\
& r_{2}=\frac{\alpha}{4}\left(u_{1}+u_{3}\right)\left(u_{2}+u_{4}\right), \\
& r_{3}=\frac{\alpha}{4}\left(u_{1}+u_{2}\right)\left(u_{3}+u_{4}\right) .
\end{aligned}
$$


It is essential that the expressions (15) and (27) for the modulus of the elliptic function for the cases $\alpha>0$ and $\alpha<0$, respectively, reduce to the same formula in terms of $r_{k}$ 's:

$$
m_{1}=m_{2}=m=\frac{r_{2}-r_{1}}{r_{3}-r_{1}},
$$

and in both cases the wavelength is given by the formula

$$
L=\frac{2 K(m)}{\sqrt{r_{3}-r_{1}}}
$$

The periodic solutions obtained in the previous section can now be written down directly in terms of $r_{j}$ 's. This would lead, however, to rather cumbersome expressions so it is better to keep the original $u_{j}$ parametrization in the periodic solutions and use the relationships (59), (60) or (63), (64) for imposing slow modulation more conveniently represented in terms of $r_{j}$ 's.

\section{The Whitham modulation equations}

The Whitham modulation equations in the Riemann form (45) can be derived using the well-established procedure of averaging the generating equation for conservation laws (see, e.g., [18]). This procedure for the Gardner equation, however, is not as straightforward as it is for the $\mathrm{KdV}$ equation, so, to avoid lengthy calculations, we make a plausible assumption that the roots $r_{j}$ of the "spectral polynomial" (55) are the Riemann invariants of the associated modulation system (45) as is the case for the related $\mathrm{KdV}$ and $\mathrm{mKdV}$ equations and other integrable systems. Then we observe that expressions (59)-(64) do agree with the corresponding $\mathrm{KdV}$ [15] and $\mathrm{mKdV}$ [23] expressions in the limits as $\alpha \rightarrow 0$ and $\alpha \rightarrow \pm \infty$, respectively. We also note that the particular set (59) of the relations between $u_{i}$ 's and $r_{i}$ 's was actually obtained (up to some obvious misprints) in [27] using the traditional finite-gap method; the remaining expressions (60), (63), and (64) have the same structure and can be simply derived as extensions of that result.

Having established the modulation Riemann invariants, the expressions for the corresponding characteristic speeds $v_{i}(\mathbf{r})$ can be derived directly from the wave-number conservation law, bypassing thus the detailed averaging procedure. To this end we consider the wave-number conservation law, which is a generic modulation equation (see, e.g., $[15,16,18]$ ),

$$
k_{t}+(k V)_{x}=0
$$

where $k(\mathbf{r})=2 \pi / L$ and $V(\mathbf{r})$ are the wave number and the phase velocity expressed in terms of the Riemann invariants. Since Eq. (68) must be consistent with the diagonal system (45), one readily obtains the potential representation

$$
v_{i}=\left(1-\frac{L}{\partial_{i} L} \partial_{i}\right) V, \quad \partial_{i} \equiv \frac{\partial}{\partial r_{i}} .
$$

The function $V(\mathbf{r})$ is given by (58) and the dependence $L(\mathbf{r})$ by (67). As a result, Eqs. (69) yield the Whitham characteristic velocities

$$
\begin{aligned}
& v_{1}=2\left(r_{1}+r_{2}+r_{3}\right)+\frac{4\left(r_{2}-r_{1}\right) K(m)}{E(m)-K(m)}, \\
& v_{2}=2\left(r_{1}+r_{2}+r_{3}\right)-\frac{4\left(r_{2}-r_{1}\right)(1-m) K(m)}{E(m)-(1-m) K(m)}, \\
& v_{3}=2\left(r_{1}+r_{2}+r_{3}\right)+\frac{4\left(r_{3}-r_{2}\right) K(m)}{E(m)},
\end{aligned}
$$

where $E(m)$ is the complete elliptic integral of the second kind. One can see that the characteristic velocities (70) coincide with those for the KdV modulation system (see, e.g., [18,32]). This, however, does not imply that the dynamics of the modulated periodic waves in the Gardner equation will necessarily be the same or even qualitatively similar to the $\mathrm{KdV}$ case. Indeed, to obtain the modulated solution for the Gardner equation, one needs first to convert the solution for $r_{i}(x, t)$ into the dynamics of the original modulation parameters $u_{i}(x, t)$ and then substitute $u_{j}$ 's into the relevant periodic solution from Sec. II (which could be drastically different from the $\mathrm{KdV}$ cnoidal wave). Moreover, since the mapping $\left\{r_{k}\right\} \mapsto\left\{u_{i}\right\}$ is not one to one, the same modulation solution $\left\{r_{k}(x, t)\right\}$ can give rise to two completely different modulations $\left\{u_{i}(x, t)\right\}$ of the periodic solutions to the Gardner equation. This becomes evident already on the level of the dispersionless limit of the Gardner equation,

$$
u_{t}+6 u(1-\alpha u) u_{x}=0,
$$

which is related to the dispersionless limit of the $\mathrm{KdV}$ equation (the Hopf equation)

$$
w_{t}+6 w w_{x}=0
$$

via the quadratic mapping $u \mapsto w$ specified by the function $w=u(1-\alpha u)$. Indeed, a constant solution $w=a$ of Eq. (72) gives rise to two different constant solutions of (71) found as roots $u^{ \pm}$of the quadratic equation $w(u)=a$ (obviously $\left.u^{+}+u^{-}=1 / \alpha\right)$. These two constant solutions can also be combined into a weak solution in the form of a propagating step: $u=u^{-}$for $x<6 a t$ and $u=u^{+}$for $x>6$ at [we note that this step solution transforms into a smooth kink (or solibore) solution (20) of the full Gardner equation if dispersion is taken into account].

It is clear that a one-to-one correspondence between the solutions of (71) and (72) is only possible in one of the restricted domains of the function $w(u): u<1 / 2 \alpha$ or $u>1 / 2 \alpha$, where $w(u)$ is monotone. Thus, one can expect significantly different, compared to the $\mathrm{KdV}$ case, dynamics if the range of the initial function $u(x, 0)$ would include an open interval containing the turning point $u=1 / 2 \alpha$ of the characteristic velocity of the dispersionless Gardner equation (71).

The above nonuniqueness in the correspondence between the modulation solutions of the $\mathrm{KdV}$ and Gardner equations is due to the invariance of the Gardner equation with respect to the transformation (5). As a result, the Whitham-Gardner system in natural modulation variables, unlike the $\mathrm{KdV}$-Whitham system, is neither genuinely nonlinear nor strictly hyperbolic (see [26] for the detailed analysis of a similar issue in the context of the closely related complex mKdV system). This results in the occurrence of much richer modulation dynamics for the Gardner equation than those for the $\mathrm{KdV}$ equation. A very similar issue was also recently discussed in [22] where the dam-break and lock-exchange flows were studied for the Miyatta-Choi-Camassa (MCC) system [36] describing fully nonlinear long dispersive interfacial waves in a two-layer fluid. This is not surprising, of course, as the Gardner equation with $\alpha>0$ represents a weakly nonlinear approximation of the MCC system obtained under an additional requirement that the layers depth ratio is close to the critical value defined by the square root of the ratio of the respective fluid densities. 
In conclusion we note that the Whitham modulation system associated with the Gardner equation with $\alpha<0$ can be elliptic (this is possible since the spectral eigenvalues $\lambda_{i}$ can be pure imaginary so the squared Riemann invariants $r_{i}$ are negative and the mapping $\left\{r_{i}\right\} \mapsto\left\{u_{i}\right\}$ is generally complex). It is indeed the case for the related focusing mKdV equation $u_{t}+6 u^{2} u_{x}+u_{x x x}=0$ (see, e.g., [23]) so modulational instability is generally to be expected in this case. However, it was shown in [37] that for the focusing $\mathrm{mKdV}$ equation with real initial data, the modulation equations arising in the zero-dispersion limit of the initial-value problem are hyperbolic which guarantees modulational stability for such problems. This property was recently used in [25] to construct undular bore solutions to the focusing $\mathrm{mKdV}$ equation. Since the focusing mKdV equation and the Gardner equation with $\alpha<0$ are related by the simple transformation (3), we shall be assuming hyperbolicity of the Gardner modulations for the resolution of a step problem considered in the next section.

\section{CLASSIFICATION OF THE SOLUTIONS FOR THE STEP PROBLEM}

We now consider the Gardner equation (1) with the initial conditions in the form of a step,

$$
u(x, 0)= \begin{cases}u^{-}, & x<0 \\ u^{+}, & x>0\end{cases}
$$

It is clear that, due to the form of the nonlinear term in the Gardner equation, the structure of the solutions to the initial value problem (1), (73) will strongly depend on the positions of the initial step parameters $u^{+}, u^{-}$relative to the turning point $u=1 / 2 \alpha$ of the dispersionless characteristic velocity $6 u(1-\alpha u)$.

\section{A. Key ingredients}

We first describe several particular solutions of the Gardner equation playing the role of "building blocks" in the full solutions to the dispersive Riemann problem (1), (73) for different values of $u^{ \pm}$. These solutions are cnoidal undular bores, rarefaction waves, solibores (for $\alpha>0$ ), and trigonometric undular bores (for $\alpha<0$ ).

(a) Cnoidal undular bores: Gurevich-Pitaevskii solution. The local structure of the simple undular bore is described by one of the periodic solutions obtained in Sec. II: solution (13) for $\alpha>0$ and solution (25) for $\alpha<0$. The corresponding modulations are expressed in terms of the parameters $r_{1}, r_{2}, r_{3}$ satisfying the Whitham equations (45), (70). It is clear that in the problem of dispersive resolution of an initial discontinuity we are interested in the similarity modulation solutions where the modulation variables depend on $s=x / t$ alone (both initial data and the modulation equations are invariant with respect to the scaling transformation $x \rightarrow C x, t \rightarrow C t$ ).

The classical Gurevich-Pitaevskii similarity solution of the modulation system (45), (70) has the form (see [17,18,32])

$$
r_{1}=r^{+}, \quad r_{3}=r^{-},
$$

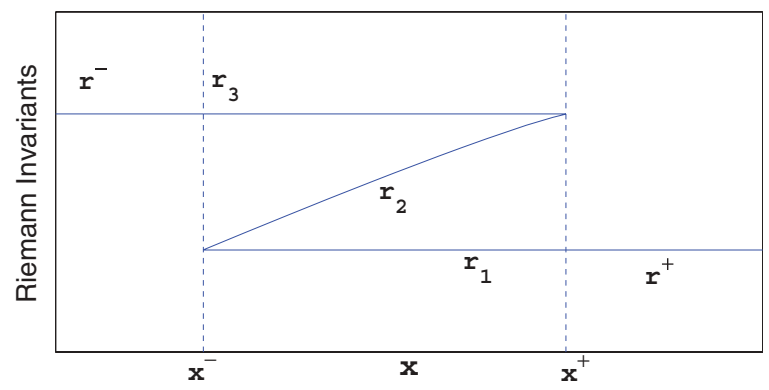

FIG. 3. (Color online) Typical behavior of the Riemann invariants $r_{j}$ in the modulation solution for a simple cnoidal undular bore.

where $r^{+}, r^{-}$are some constants, while the dependence $r_{2}(x, t)$ is given implicitly by

$$
v_{2}\left(r^{+}, r_{2}, r^{-}\right)=\frac{x}{t} .
$$

It is essential that, since $r_{3}>r_{1}$, one must have

$$
r^{-}>r^{+} \text {. }
$$

A typical modulation solution is presented in Fig. 3.

The undular bore described by the Gurevich-Pitaevskii modulation solution (74), (75) occupies an expanding region $x^{-}<x<x^{+}$whose edges $x^{ \pm}=s^{ \pm} t$ propagate with constant velocities $s^{ \pm}$. The trailing (harmonic) edge is defined by the condition $m=0$ [i.e., $r_{2}=r_{1}=r^{+}$, see (66)] and the leading (soliton) edge-by the condition $m=1$ (i.e., $r_{2}=r_{3}=r^{-}$). Then the velocities $s^{ \pm}$are found from (75), (74), (70) as

$$
\begin{aligned}
& s^{-}=\left.v_{2}\right|_{r_{2}=r_{1}}=12 r_{1}-6 r_{3}=12 r^{+}-6 r^{-}, \\
& s^{+}=\left.v_{2}\right|_{r_{2}=r_{3}}=2 r_{1}+4 r_{3}=2 r^{+}+4 r^{-} .
\end{aligned}
$$

The above solution (74), (75) coincides with the modulations in the undular bore arising as a result of the resolution of an initial discontinuity: $r(x>0,0)=r^{+}$and $r(x<0,0)=r^{-}$ for the $\mathrm{KdV}$ equation $r_{t}+6 r r_{x}+r_{x x x}=0$ [17]. However, in the $\mathrm{KdV}$ context the modulation solution (74), (75) uniquely characterizes the asymptotic solution for $\mathrm{KdV}$ undular bore due to the one-to-one correspondence between the Riemann invariants $\left\{r_{j}\right\}$ and the physical parameters $\left\{u_{j}\right\}$ of the traveling wave solution [15]. In the case of the Gardner equation (1) there are two possibilities for each sign of $\alpha$ due to different possible relationships between the $\left\{r_{k}\right\}$ and $\left\{u_{i}\right\}$ for different types of traveling wave solutions described in Sec. III B-see relationships (59), (60) for $\alpha>0$ and (63), (64) for $\alpha<0$. The actual choice depends on the positions of the initial step parameters $u^{+}$and $u^{-}$relative to the turning point $u=1 /(2 \alpha)$ of the function $w(u)=u(1-\alpha u)$ (see Sec. III C). We shall consider all possible cases in the next two sections.

(b) Rarefaction waves. The rarefaction waves are asymptotically described by the similarity solution

$$
\frac{x}{t}=6 u(1-\alpha u)
$$

of the dispersionless limit (71) of the Gardner equation. The two possible roots of (79) describe two types of rarefaction waves: "normal" and "reverse." We first consider the case $\alpha>0$. 
The normal simple rarefaction wave connects two constant states $u=u^{l}$ (left) and $u=u^{r}$ (right) satisfying the condition $u^{l}<u^{r}<1 / 2 \alpha$ and has a structure similar to the rarefaction solution of the "rightward-propagating" $\mathrm{KdV}$ equation, in which $\partial u / \partial x>0$. It is described by the equation

$$
\begin{aligned}
& u=u^{l} \quad \text { for } x<s^{l} t, \\
& u=\frac{1}{2 \alpha}\left(1-\sqrt{1-\frac{2 \alpha x}{3 t}}\right) \quad \text { for } s^{l} t<x<s^{r} t, \\
& u=u^{r}, \quad \text { for } x>s^{r} t .
\end{aligned}
$$

We shall be using a symbolic diagram $\left\{u^{l} \mathbf{R W} \rightarrow u^{r}\right\}$ for the normal rarefaction wave.

The reversed simple rarefaction wave connects two constant states $u=u^{l}$ and $u=u^{r}$ satisfying the condition $u^{r}<u^{l}<$ $1 / 2 \alpha$ and is described by the equation

$$
\begin{aligned}
& u=u^{l} \quad \text { for } x<s^{l} t, \\
& u=\frac{1}{2 \alpha}\left(1+\sqrt{1-\frac{2 \alpha x}{3 t}}\right) \text { for } s^{l} t<x<s^{r} t, \\
& u=u^{r}, \quad \text { for } x>s^{r} t .
\end{aligned}
$$

The reversed rarefaction wave is similar to the rarefaction wave in the "leftward-propagating" $\mathrm{KdV}$ equation, in which $\partial u / \partial x<0$. The symbolic diagram for this wave is $\left\{u_{l} \leftarrow \mathbf{R W} u^{r}\right\}$.

The speeds $s^{l, r}$ of the left and right boundaries for both normal and reversed rarefaction waves are given by

$$
s^{l, r}=6 u^{l, r}\left(1-\alpha u^{l, r}\right), \quad s^{l}<s^{r} .
$$

Solutions (81) and (80) have weak discontinuities at the corners $x=s^{l, r} t$. These are smoothed out by small-amplitude oscillatory wave trains which are generated if the dispersive term of the Gardner equation is taken into account (cf. [32] for the $\mathrm{KdV}$ case).

For $\alpha<0$, the rarefaction waves are described by the same solutions, however, formula (80) would then describe the reversed wave with $u^{r}<u^{l}<-1 /(2|\alpha|)$ and (81)-the normal one with $u^{r}>u^{l}>-1 /(2|\alpha|)$.

(c) Solibores $(\alpha>0)$. The solibore (kink) solutions for the Gardner equation with $\alpha>0$ are given by formulas (20). Solibores provide the smooth transition between two constant states $u^{l}$ and $u^{r}$ satisfying the condition $u^{l}+u^{r}=1 / \alpha$. Using the terminology introduced above for rarefaction waves, we shall refer to the solibore as normal when we have $\partial u / \partial x<0$ ["-" sign in solution (20)] and reverse, when $\partial u / \partial x>0["+"$ sign in (20)]. The corresponding diagrams are $\left\{u^{l} \mathbf{S B} \rightarrow u^{r}\right\}$ for the normal solibore and $\left\{u^{l} \leftarrow \mathbf{S B} u^{r}\right\}$ for the the reversed one.

(d) Trigonometric undular bores $(\alpha<0)$. This type of undular bores, not encountered in the KdV theory, was first reported in [25] where the evolution of a step problem was studied for the focusing $\mathrm{mKdV}$ equation (see also a similar solution for the complex modified $\mathrm{mKdV}$ equation in [26]). The trigonometric undular bores of the Gardner equation with $\alpha<0$ are described by the modulated finiteamplitude nonlinear periodic solutions (31) or (41) so that $m=0$ throughout the wave train. At one of the edges of the trigonometric bore the amplitude vanishes and at the opposite edge it assumes some finite value. Generically,

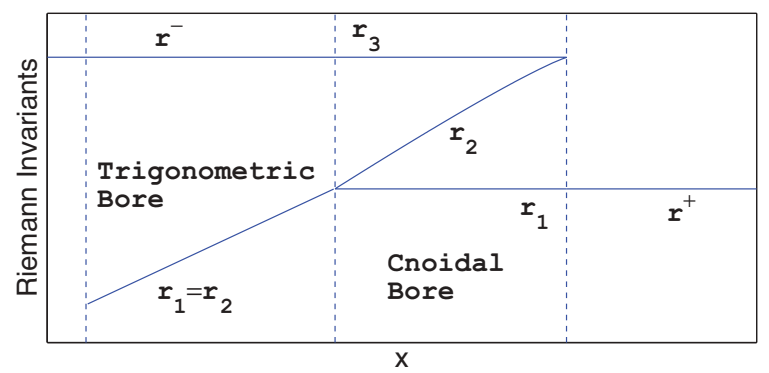

FIG. 4. (Color online) The sketch of the "square" Riemann invariants $r_{1}, r_{2}, r_{3}$ behavior in the composite cnoidal-trigonometric undular bore.

as will be explained later, trigonometric undular bores are realized as parts of composite solutions (either a combination of cnoidal and trigonometric bores or a combination of a trigonometric bore and a rarefaction wave). As with other wave patterns arising for the Gardner equation, one can have two types of trigonometric bores: normal, $\left\{u^{l} \mathbf{T B} \rightarrow u^{r}\right\}$ and reversed, $\left\{u^{l} \leftarrow \mathbf{T B} u^{r}\right\}$. The normal trigonometric bore is locally described by solution (41) while for the reversed one solution (31) should be used.

A typical configuration of the Riemann invariants $r_{j}$ in the composite cnoidal-trigonometric bore is shown in Fig. 4. Since in the region of the trigonometric bore one has $r_{2}=r_{1}=r$, the corresponding similarity modulation solution $v_{2}\left(r, r, r^{-}\right)=$ $x / t$ is degenerate in the sense that it does not allow one to reconstruct uniquely the modulations for $u_{j}(x, t)$ in the trigonometric bore and to provide the necessary matching between $u^{l}$ and $u^{r}$ across the bore. Therefore, the "square" Riemann invariants $r_{j}$ (62) used so far, are not suitable for the description of trigonometric bores. Instead, motivated by the results in [25], we introduce the classical Whitham combinations

$$
\begin{aligned}
& R_{1}=\frac{1}{2}\left(u_{2}+u_{3}\right)=\frac{1}{2 \alpha}\left(1+\sqrt{1-4 \alpha r_{1}}\right), \\
& R_{2}=\frac{1}{2}\left(u_{2}+u_{4}\right)=\frac{1}{2 \alpha}\left(1-\sqrt{1-4 \alpha r_{2}}\right), \\
& R_{3}=\frac{1}{2}\left(u_{3}+u_{4}\right)=\frac{1}{2 \alpha}\left(1-\sqrt{1-4 \alpha r_{3}}\right)
\end{aligned}
$$

$\left(R_{3} \geqslant R_{2} \geqslant R_{1}\right.$ ) for the normal trigonometric undular bore [see the relationships (64)] and

$$
\begin{aligned}
& R_{3}=\frac{1}{2}\left(u_{1}+u_{2}\right)=\frac{1}{2 \alpha}\left(1+\sqrt{1+4 \alpha r_{3}}\right), \\
& R_{2}=\frac{1}{2}\left(u_{1}+u_{3}\right)=\frac{1}{2 \alpha}\left(1+\sqrt{1+4 \alpha r_{2}}\right), \\
& R_{1}=\frac{1}{2}\left(u_{2}+u_{3}\right)=\frac{1}{2 \alpha}\left(1-\sqrt{1-4 \alpha r_{1}}\right)
\end{aligned}
$$

$\left(R_{1} \geqslant R_{2} \geqslant R_{3}\right.$ ) for the reversed trigonometric undular bore [see the relationships (63)].

Obviously, the quantities $R_{j}\left(r_{j}\right)$ are the Riemann invariants of the modulation system (any function of the Riemann invariant alone is also a Riemann invariant).

(i) Normal trigonometric bores. We now construct the modulation solution for the normal trigonometric bore, where the oscillations occur between the roots $u_{3}$ and $u_{4}$ of the traveling wave solution polynomial $Q(u)(8)$. The am- 
plitude in such a bore is

$$
a=u_{4}-u_{3}=2\left(R_{2}-R_{1}\right)
$$

and it gradually increases from $a=0$ at the left (harmonic) edge, say $x=x^{l}$, to some nonzero value $a=a^{r}$ at the right edge $x=x^{r}$.

When $a=0$ we have from (41) $u=u_{3}=u_{4}$. Hence, since the trigonometric bore must match with $u=u^{l}$ at $x=x^{l}$ we obtain from (83)

$$
R_{3}=u_{4}=u^{l} \quad \text { at } x=x^{l},
$$

and, therefore, $R_{3}=u^{l}$ everywhere within the trigonometric bore. Hence, from (83) we obtain

$$
r_{3}=u^{l}\left(1-\alpha u^{l}\right) \text {. }
$$

Now, within the trigonometric undular bore we have $r_{1}=$ $r_{2}=r(x / t)$, which is determined by the degenerate similarity solution of the Whitham system (45),

$$
\left.v_{2}\right|_{m=0}=12 r-6 r_{3}=\frac{x}{t} \text {. }
$$

Substituting the value of $r_{3}$ (87) we obtain

$$
r=\frac{1}{12}\left[\frac{x}{t}+6 u^{l}\left(1-\alpha u^{l}\right)\right],
$$

and so from (83) we get

$$
\begin{aligned}
& R_{1}=\frac{1}{2 \alpha}\left\{1+\sqrt{2 \alpha^{2}\left(u^{l}-\frac{1}{2 \alpha}\right)^{2}+\frac{1}{2}\left(1-\frac{2 \alpha x}{3 t}\right)}\right\}, \\
& R_{2}=\frac{1}{2 \alpha}\left\{1-\sqrt{2 \alpha^{2}\left(u^{l}-\frac{1}{2 \alpha}\right)^{2}+\frac{1}{2}\left(1-\frac{2 \alpha x}{3 t}\right)}\right\} .
\end{aligned}
$$

One can see that $R_{1}+R_{2}=1 / \alpha$ for all $x$ in the trigonometric bore.

If a trigonometric undular bore is fully realized (i.e., is not part of the composite cnoidal-trigonometric bore) then at the leading edge $x=x^{r}$ it must assume the limiting wave form of a bright algebraic soliton (43) (otherwise the matching with constant or smooth external solution would not be possible). This implies that we have $u_{3}=u_{2}=u_{1}$ and so $R_{2}=R_{3}=u^{l}$ at the leading edge $x=x^{r}$. The algebraic soliton rides on the background $u_{1}$ so the relevant matching condition becomes

$$
u_{1}=u^{r} \quad \text { at } x=x^{r},
$$

which, by $u_{1}=u_{2}=u_{3}$ and the first formula in (83) implies $R_{1}=u^{r}$ at $x=x^{r}$. Therefore, the trigonometric bore can only connect the states $u^{l}$ and $u^{r}$ satisfying the condition

$$
u^{l}+u^{r}=\frac{1}{\alpha}
$$

i.e., a single isolated trigonometric bore can be realized as a result of the step evolution only in the special cases when the parameters of the initial step satisfy the condition (92) (note that for normal trigonometric bore one must have $u^{l}>$ $u^{r}$ which follows from the ordering $R_{3}>R_{1}$ ). Thus, for the Gardner equation with $\alpha<0$ the trigonometric undular bores play the role similar to that played by solibores in the step problem for the Gardner equation with $\alpha>0$.
The speed $s^{l}$ of the trailing edge of the trigonometric bore is found from the condition that at the trailing edge $R_{2}=R_{1}=$ $\frac{1}{2 \alpha}$ which implies by (90)

$$
s^{l}=\frac{3}{\alpha}-6 \alpha u^{l}\left(1-\alpha u^{l}\right) .
$$

At the leading edge $x=s^{r} t$ of the trigonometric undular bore we have $R_{2}=R_{3}=u^{l}$. Then, substituting $R_{2}=u^{l}, x / t=s^{r}$ into the second equation (90) we obtain the speed of the leading edge

$$
s^{r}=6 u^{l}\left(1-\alpha u^{l}\right)=6 u^{r}\left(1-\alpha u^{r}\right),
$$

which coincides with the characteristic speed of the dispersionless Gardner equation at $u=u^{r}$. This implies, in particular, that the trigonometric undular bore can be joined at the leading edge to a simple rarefaction wave solution.

The amplitude of the algebraic soliton (43) at the leading edge follows from (85) where we set $R_{2}=R_{3}$ :

$$
a^{r}=\left.2\left(R_{3}-R_{1}\right)\right|_{x=x^{r}}=2\left(u^{l}-u^{r}\right)=2\left(2 u^{l}-1 / \alpha\right) .
$$

(ii) Reversed trigonometric bores. In the reversed trigonometric bore the oscillations occur between the roots $u_{1}$ and $u_{2}$ of the polynomial $Q(u)$ in (8). The amplitude is given by

$$
a=u_{2}-u_{1}=2\left(R_{1}-R_{2}\right) .
$$

In terms of the Riemann invariants $r_{j}$ the modulation solution for the reversed bore is given by the same formula (89), which is then translated to $R_{j}$ 's (84) as [cf. (90)]

$$
\begin{aligned}
& R_{3}=u^{l} \\
& R_{2}=\frac{1}{2 \alpha}\left\{1+\sqrt{2 \alpha^{2}\left(u^{l}-\frac{1}{2 \alpha}\right)^{2}+\frac{1}{2}\left(1-\frac{2 \alpha x}{3 t}\right)}\right\} \\
& R_{1}=\frac{1}{2 \alpha}\left\{1-\sqrt{2 \alpha^{2}\left(u^{l}-\frac{1}{2 \alpha}\right)^{2}+\frac{1}{2}\left(1-\frac{2 \alpha x}{3 t}\right)}\right\}
\end{aligned}
$$

Similar to the normal trigonometric bore, the reversed trigonometric bore has a restriction (92) for the admissible boundary values $u^{l}$ and $u^{r}$. At the leading edge $x=x^{r}$ the reversed bore assumes the limiting form of a dark algebraic soliton (33), which has the amplitude $a^{r}=2\left(2 u^{l}-1 / \alpha\right)$. The trailing and the leading edge speeds are given by the same expressions (93) and (94), respectively.

As was already mentioned, the trigonometric undular bore (normal or reversed) can occur as part of the composite cnoidal-trigonometric bore. In that case it is realized only partially and does not contain an algebraic soliton at the leading edge. The two bores match at the trailing edge of the cnoidal bore, which is defined by (77) with $r^{-}=u^{l}\left(1-\alpha u^{l}\right)$ and $r^{+}=u^{r}\left(1-\alpha u^{r}\right)$

$$
s^{*}=12 u^{r}\left(1-\alpha u^{r}\right)-6 u^{l}\left(1-\alpha u^{l}\right) .
$$

The amplitude at the matching point for the normal composite bore is [see (85), (90)]

$$
a^{*}=2\left[R_{2}\left(s^{*}\right)-R_{1}\left(s^{*}\right)\right]=4\left|\frac{1}{2 \alpha}-u^{r}\right| .
$$




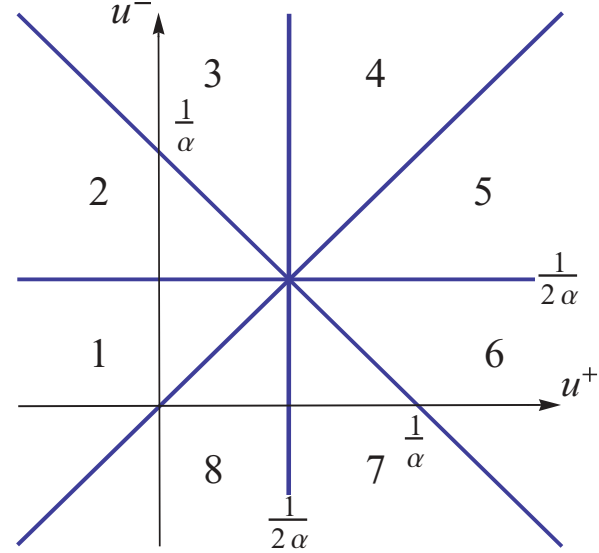

FIG. 5. (Color online) Parametric map of solutions of the step problem for the Gardner equation with $\alpha>0$. The resolution diagrams corresponding to each of the cases on the plane of the initial step parameters $u^{-}$and $u^{+}$are the following: Region 1: $\left\{u^{-} \mathbf{U B} \rightarrow u^{+}\right\}$; Region 2: $\left\{u^{-} \leftarrow \mathbf{U B}\left(u^{*}\right) \mathbf{S B} \rightarrow u^{+}\right\}$; Region 3: $\left\{u^{-} \leftarrow \mathbf{R W}\left(u^{*}\right) \mathbf{S B} \rightarrow u^{+}\right\}$; Region $4:\left\{u^{-} \leftarrow \mathbf{R W} u^{+}\right\}$; Region 5: $\left\{u^{-} \leftarrow \mathbf{U B} u^{+}\right\}$; Region 6: $\left\{u^{-} \mathbf{U B} \rightarrow\left(u^{*}\right) \leftarrow \mathbf{S B} u^{+}\right\}$; Region 7: $\left\{u^{-}\right.$ $\left.\mathbf{R W} \rightarrow\left(u^{*}\right) \leftarrow \mathbf{S B} u^{+}\right\}$; Region 8: $\left\{u^{-} \mathbf{R W} \rightarrow u^{+}\right\}$. In all relevant cases the intermediate state $u^{*}=1 / \alpha-u^{+}$.

The same result obviously holds for the reversed composite bore, for which we use (96) and (97). The trailing edge speeds for both types of composite bores is given by (93).

\section{B. Classification for $\alpha>0$}

We can now proceed with the full classification of the solutions to the step problem.

We first present a detailed classification of solutions to the evolution of a step problem for the Gardner equation with $\alpha>0$. The parametric map of solutions is constructed on the $\left(u^{+}, u^{-}\right)$plane of the initial step parameters (see Fig. 5). The whole $\left(u^{-}, u^{+}\right)$plane is split into eight regions, each corresponding either to one of the basic patterns listed in Sec. IV A ("pure" solutions) or to the combination of two patterns ("composite" solutions). To represent the result of the evolution of an initial step for each region we shall be using symbolical diagrams introduced in the previous section. Say, the resolution diagram for Region $6,\left\{u^{-} \mathbf{U B} \rightarrow\left(u^{*}\right) \leftarrow \mathbf{S B}\right.$ $\left.u^{+}\right\}$, denotes a normal undular bore connecting the left state $u^{-}$with an intermediate state $u^{*}$ which is further connected to the right state $u^{+}$via the reversed solibore.

The lines separating the regions are

(a) $u^{-}=u^{+}$-separates the regions of pure undular bores and pure rarefaction waves;

(b) $u^{-}=1 / \alpha-u^{+}$-corresponds to the steps resolving into single solibores and separates the regions of composite solutions of different types: undular bore + solibore and rarefaction wave + solibore; and

(c) $u^{-}=1 / 2 \alpha$ - separates regions of pure (undular bore) and composite (undular bore + solibore) solutions.

We note that the classification for $\alpha>0$ is qualitatively similar to that presented in [22] for the MCC system describing fully nonlinear interfacial dispersive waves in a two-layer fluid. In [22], the analytic method of [20] was used to obtain the locations of undular bore boundaries and the leading solitary wave amplitude. However, the full modulation solutions are not available for the MCC system due to complexity of the corresponding Whitham equations. The theory presented in this paper has an obvious advantage of greater simplicity and universality due to the integrable nature of the problem and availability of exact analytic solutions. At the same time, in the context of internal water waves, the Gardner equation with $\alpha>0$, being a weakly nonlinear approximation of the MCC system, is quantitatively valid only for the waves of sufficiently small amplitude.

The classification is most conveniently performed using the function $w(u)=u(1-\alpha u)$ defining the mapping from the dispersionless Gardner equation (71) to the dispersionless limit of the KdV equation [the Hopf equation (72); see Sec. III C]. We shall illustrate each wave pattern in the classification by presenting the analytical (modulation theory) solutions along with respective direct numerical solutions of the Gardner equation. In our numerics, Eq. (1) was solved using the method of lines (see, e.g., [38]) where the spatial derivatives are discretized using second-order accurate finite difference approximation to reduce the governing partial differential equation to a system of ordinary differential equations. This system is then solved using the fourth-order Runge-Kutta method.

Region $1, u^{+}<u^{-} \leqslant \frac{1}{2 \alpha},\left\{u^{-} \mathbf{U B} \rightarrow u^{+}\right\}$. Both values $u^{-}$and $u^{+}$lie in the domain where the function $w(u)=$ $u(1-\alpha u)$ is monotonically increasing so there is one-toone correspondence between the dispersionless limits of the Gardner and the $\mathrm{KdV}$ equations. This suggests that in the Region 1 initial discontinuity can be resolved by a single normal "shallow-water" undular bore of the KdV type with the bright soliton at the leading edge and the linear wave packet at the trailing edge.

We shall use the traveling wave solution (13) and the relationships (59) between $u_{j}$ 's and $r_{i}$ 's to construct the desired modulated traveling wave solution for the undular bore and show that it indeed provides the required matching between $u^{-}$and $u^{+}$. The parameters $r^{ \pm}$entering the modulation solution (70), (75) can be expressed in terms of the initial step parameters $u^{ \pm}$using the relationships (59).

It follows for the small-amplitude limit (21) of the traveling wave solution (13) that the trailing edge of the undular bore $(m=0)$ propagates against the background $u=u_{2}=u_{3}$ [this can be inferred directly from the ordinary differential equation (8), where we set $u_{2} \rightarrow u_{3}$; see Fig. 1(e)]. Similarly, for the soliton edge $m \rightarrow 1$ we have that the leading bright soliton propagates on the background $u=u_{1}=u_{2}$ [see (18) and Fig. 1(b)]. Thus, if the step is resolved by a single undular bore we must require

$$
u_{2}=u_{3}=u^{-}
$$

at the trailing edge and

$$
u_{2}=u_{1}=u^{+}
$$

at the leading edge.

Considering the same limits in the relationships (59) we have

$$
\left.u_{2}\right|_{m=0}=\left.u_{3}\right|_{m=0}=\frac{1}{2 \alpha}\left(1-\sqrt{1-4 \alpha r_{3}}\right)
$$


and

$$
\left.u_{1}\right|_{m=1}=\left.u_{2}\right|_{m=1}=\frac{1}{2 \alpha}\left(1-\sqrt{1-4 \alpha r_{1}}\right) .
$$

According to (74) $r_{3}=r^{-}$and $r_{1}=r^{+}$in the GurevichPitaevskii solution. Then, from (100)-(103) we have

$$
\begin{aligned}
& r^{-}=u^{-}\left(1-\alpha u^{-}\right)=w\left(u^{-}\right), \\
& r^{+}=u^{+}\left(1-\alpha u^{+}\right)=w\left(u^{+}\right) .
\end{aligned}
$$

Since for the considered Region 1 we have $w\left(u^{-}\right)>w\left(u^{+}\right)$, the condition (76) of the Riemann invariant ordering is satisfied, and therefore our construction is consistent throughout.

The undular bore occupies the region $s^{-} t<x<s^{+} t$ where the edge speeds $s^{ \pm}$are obtained from (77) and (78),

$$
\begin{aligned}
& s^{-}=12 u^{+}\left(1-\alpha u^{+}\right)-6 u^{-}\left(1-\alpha u^{-}\right), \\
& s^{+}=2 u^{+}\left(1-\alpha u^{+}\right)+4 u^{-}\left(1-\alpha u^{-}\right) .
\end{aligned}
$$

The width of the undular bore is then

$$
\begin{aligned}
\Delta & =\left(s^{+}-s^{-}\right) t=10\left(r^{-}-r^{+}\right) t \\
& =10\left(u^{-}-u^{+}\right)\left[1-\alpha\left(u^{-}+u^{+}\right)\right] t .
\end{aligned}
$$

One can see that the Gardner undular bore is narrower than its $\mathrm{KdV}$ counterpart, for which one has $\Delta_{\mathrm{KdV}}=10\left(u^{-}-u^{+}\right) t$ for the same initial conditions. As a matter of fact the KdV result is reproduced when $\alpha=0$. The amplitude of the lead soliton in the undular bore is [see (18), (59), and (104)]

$$
a^{+}=\left.\left(u_{3}-u_{1}\right)\right|_{m=1}=2\left(u^{-}-u^{+}\right) .
$$

This result coincides with the classical KdV formula for the lead soliton amplitude.

The constructed solution is illustrated in Fig. 6 where the plot (a) of the analytical (modulation theory) solution is presented along with the direct numerical solution of the Gardner equation [plot (b)]. One can see that agreement is very good. The presence in the numerical plot of an extended smallamplitude oscillatory tail stretching behind the trailing edge as defined by the modulation theory is a well-known feature of undular bore solutions observed in the early comparisons of the KdV modulation solutions with numerics (see, e.g., [39]) and recently studied in detail in [40].

Region $2, u^{+}<\frac{1}{2 \alpha}<u^{-}<1 / \alpha-u^{+},\left\{u^{-} \leftarrow \mathbf{U B}\left(u^{*}\right) \mathbf{S B}\right.$ $\rightarrow u^{+}$. Since $u^{+}$and $u^{-}$now lie in different regions of monotonicity of the function $w(u)=u(1-\alpha u)$, the Region 1 solution in the form of a single normal undular bore is not able to provide the necessary continuous matching between the given states. Instead, a reversed undular bore is generated between $u^{-}$and the intermediate state $u^{*}=\left(1 / \alpha-u^{+}\right)>u^{-}$. This intermediate state is found from the condition $w\left(u^{*}\right)=$ $w\left(u^{+}\right)$and corresponds to the required boundary value for the Riemann invariant, $r^{+}=u^{+}\left(1-\alpha u^{+}\right)$but lies in the same as $u^{+}$region of monotonicity of the mapping function $w(u)$ [see Fig. 7(a)]. The further connection between $u^{*}$ and $u^{+}$ is provided by a normal solibore. The corresponding wave pattern is presented in Fig. 8 .

The relationship between the Riemann invariants $\left\{r_{i}\right\}$ and the undular bore parameters $\left\{u_{i}\right\}$ is now described by formulas (60). The trailing edge $(m=0)$ of the bore propagates on a background $u=u_{3}=u_{2}=u^{-}$. At the leading edge
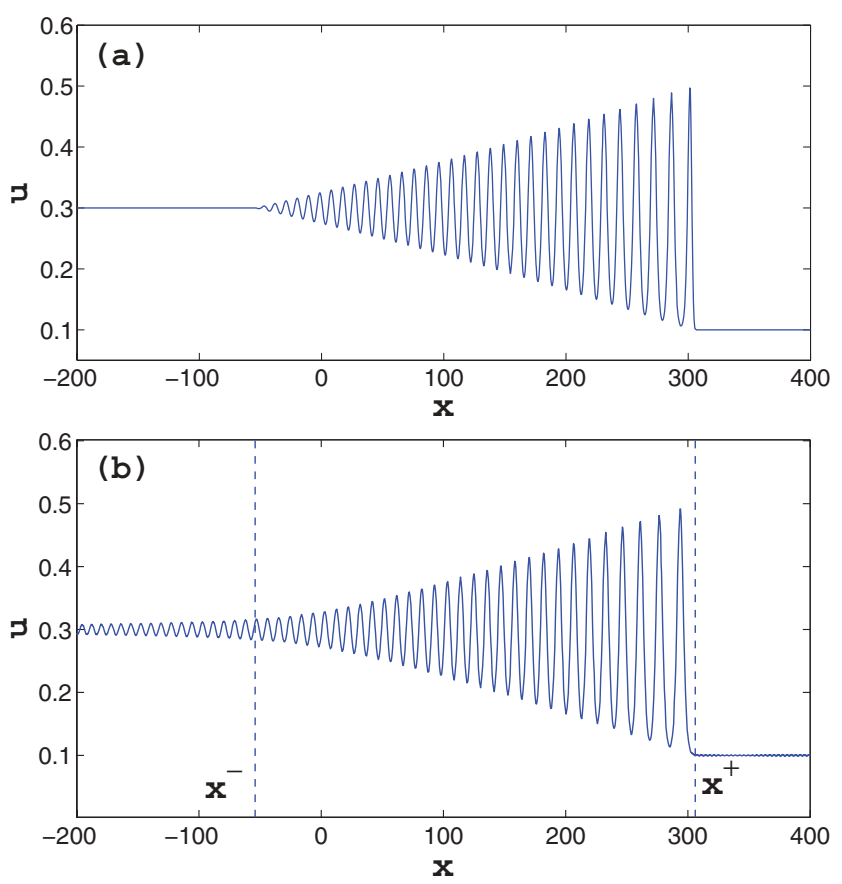

FIG. 6. (Color online) Evolution of an initial discontinuity for the Gardner equation with $\alpha=1$. Region $\left.1:\left\{u^{-} \mathbf{U B} \rightarrow u^{+}\right\}\right)$. The step parameters are $u^{-}=0.3, u^{+}=0.1$. (a) Analytical solution in the form of a modulated periodic wave. (b) Numerical solution; the analytically found edges $x^{ \pm}=s^{ \pm} t$ of the undular bore are shown by dashed lines. Both plots correspond to $t=300$.

( $m=1)$ we have a dark soliton (19) propagating against the background $u=u_{3}=u_{4}$. Thus, for the undular bore we have

$$
u_{3}=u_{2}=u^{-}
$$
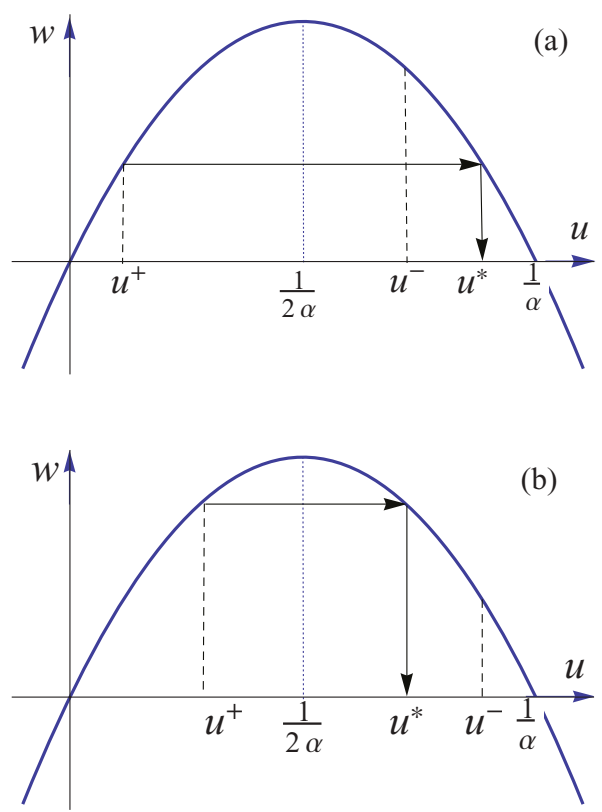

FIG. 7. (Color online) Finding the intermediate state $u=u^{*}$. (a) Region 2 diagram, $u^{*}>u^{-}$. (b) Region 3 diagram, $u^{*}<u^{-}$. 

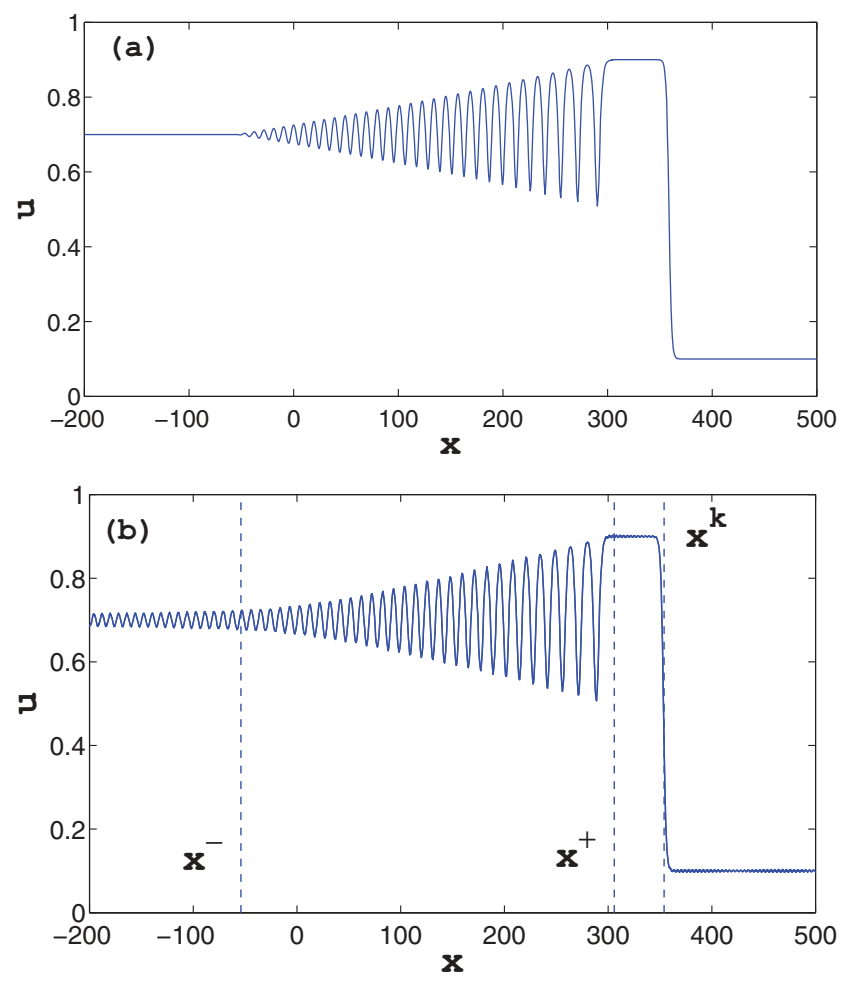

FIG. 8. (Color online) Evolution of an initial discontinuity for the Gardner equation with $\alpha=1$. Region $2,\left\{u^{-} \leftarrow \mathbf{U B}\left(u^{*}\right) \mathbf{S B} \rightarrow u^{+}\right\}$. The initial step parameters are $u^{-}=0.7, u^{+}=0.1$. (a) Analytical solution in the form of a modulated periodic wave connected to a solibore. (b) Numerical solution of the Gardner equation; the analytically found edges $x^{ \pm}=s^{ \pm} t$ and $x^{k}=s^{k} t$ of the undular bore and solibore, respectively, are shown by dashed lines. Both plots correspond to $t=300$.

and

$$
u_{3}=u_{4}=u^{*}=\frac{1}{\alpha}-u^{+} .
$$

Then from (60) we obtain

$$
\left.u_{2}\right|_{m=0}=\left.u_{3}\right|_{m=0}=\frac{1}{2 \alpha}\left(1+\sqrt{1-4 \alpha r_{3}}\right)
$$

and

$$
\left.u_{3}\right|_{m=1}=\left.u_{4}\right|_{m=1}=\frac{1}{2 \alpha}\left(1+\sqrt{1-4 \alpha r_{1}}\right) .
$$

Again, since $r_{1}=r^{+}$and $r_{3}=r^{-}$in the modulation solution for the undular bore, we have from (108)-(111)

$$
\begin{aligned}
& r_{3}=r^{-}=u^{-}\left(1-\alpha u^{-}\right)=w\left(u^{-}\right), \\
& r_{1}=r^{+}=u^{*}\left(1-\alpha u^{*}\right)=u^{+}\left(1-\alpha u^{+}\right) .
\end{aligned}
$$

Thus, the expressions for the undular bore speeds remain the same [cf. (105)]:

$$
\begin{aligned}
& s^{-}=12 u^{+}\left(1-\alpha u^{+}\right)-6 u^{-}\left(1-\alpha u^{-}\right), \\
& s^{+}=2 u^{+}\left(1-\alpha u^{+}\right)+4 u^{-}\left(1-\alpha u^{-}\right) .
\end{aligned}
$$

The front solibore connecting the states $u^{*}$ and $u^{+}$is described by formula (20). The solibore speed is given by

$$
s^{k}=\frac{1}{\alpha}+2 \alpha u^{+} u^{*}=\frac{1}{\alpha}+2 u^{+}\left(1-\alpha u^{+}\right) .
$$
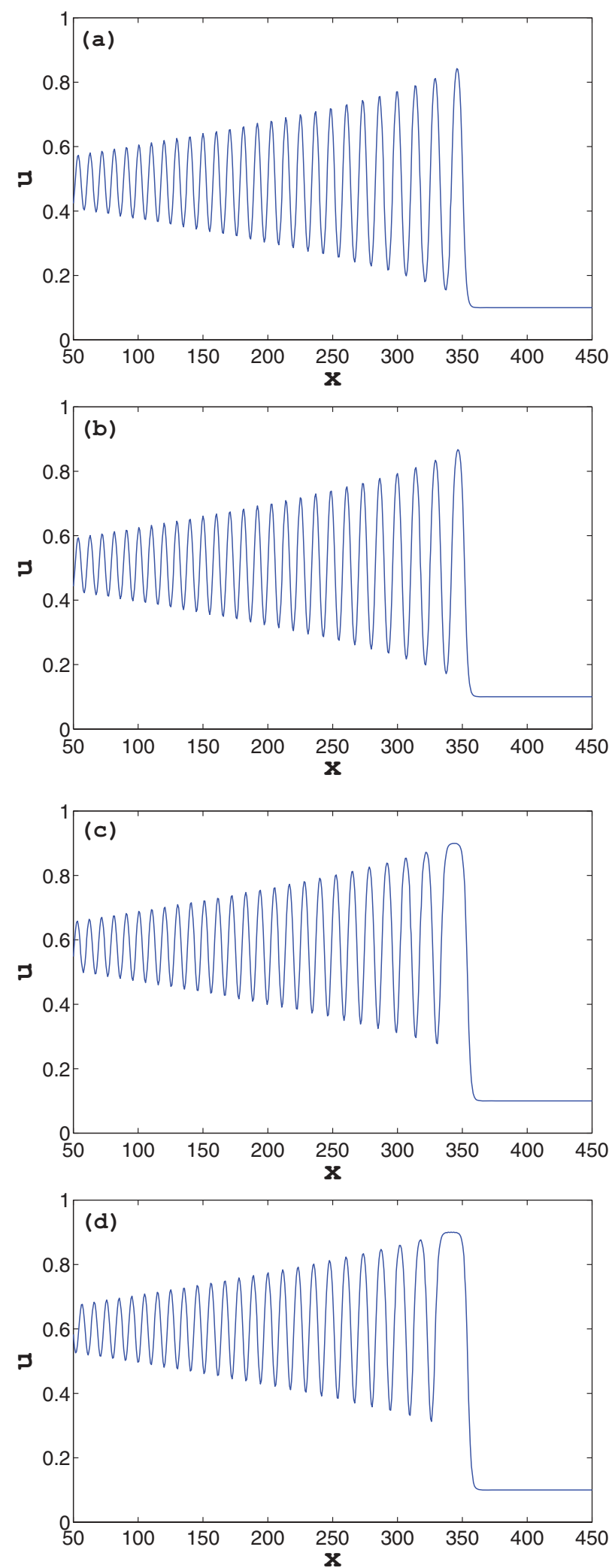

FIG. 9. (Color online) The transformation of the undular bore structure from the normal, "bright soliton" pattern in Region 1 [plot (a)] to the reversed, "dark soliton plus solibore" pattern in Region 2 [plot (d)]. Numerical simulations of the Gardner equation with $\alpha=1$. The downstream state, $u^{+}=0.1$, is the same for all cases; the upstream state $u^{-}$is taken in the range $u^{-}=0.49<1 / 2 \alpha$ (Region 1) to $u^{-}=0.58>1 / 2 \alpha($ Region 2$), t=300$. 

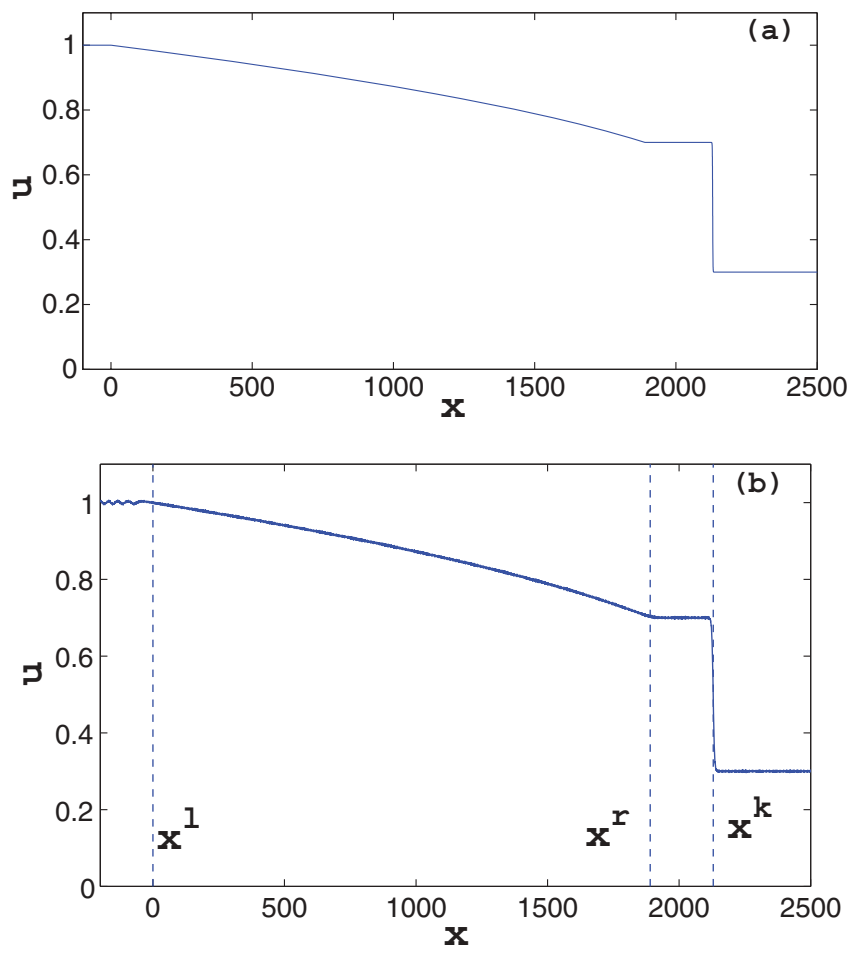

FIG. 10. (Color online) Evolution of an initial discontinuity for the Gardner equation with $\alpha=1$. Region 3: $\left\{u^{-} \leftarrow \mathbf{R W}\left(u^{*}\right) \mathbf{S B} \rightarrow\right.$ $\left.u^{+}\right\}$. The initial step parameters are $u^{-}=1.0, u^{+}=0.3$. (a) Analytical (dispersionless limit) solution. (b) Numerical solution of the Gardner equation. Dashed lines in (b) correspond to the analytically found locations of the rarefaction wave edges $x^{l, r}=s^{l, r} t$ and solibore position $x^{k}=s^{k} t$. Both plots are made for $t=1500$.

Now it is not difficult to see that

$$
s^{k}-s^{+}=4 \alpha\left(u^{-}-\frac{1}{2 \alpha}\right)^{2} \geqslant 0 ;
$$

that is, the solibore always propagates ahead of the undular bore as expected. Since there is a qualitative change of the wave pattern in the transition from Region 1 to Region 2 it is necessary to look closer at what happens near the boundary between these two regions determined by the value of the left state $u^{-}=1 / 2 \alpha$. The change of the pattern is illustrated in Fig. 9 where several numerical solutions of the Gardner equation with $\alpha=1$ are presented for the evolution of initial discontinuities with the same right state, $u^{+}=0.1$, while the left state $u^{-}$was taken in the range $u^{-}=0.49<1 / 2 \alpha$ (Region 1) to $u^{-}=0.58>1 / 2 \alpha$ (Region 2).

One can see from (115) that for $u^{-}=1 / 2 \alpha$ the speed of the solibore coincides with the speed of the leading soliton in the undular bore so the "borderline" wave pattern in Fig. 9(b) can be interpreted in both ways: as a normal, bright, undular bore or, equivalently, as a reversed, dark, undular bore with an attached solibore. When we increase $u^{-}$, the solibore separates from the undular bore, which in its turn acquires the reversed wave form with a distinct dark soliton structure near the leading edge [see Fig. 9(d)].

Region 3, $1 / \alpha-u^{-}<u^{+}<\frac{1}{2 \alpha}<u^{-}, u^{-}+u^{+}>1 / \alpha$, $\left\{u^{-} \leftarrow \mathbf{R W}\left(u^{*}\right) \mathbf{S B} \rightarrow u^{+}\right\}$. This region is analogous to Region 2 , since the values $u^{-}$and $u^{+}$again lie in different do- mains of monotonicity of the function $w(u)$, thus a single-wave resolution is not possible. However, now the intermediate state satisfies the inequality $u^{*}=1 / \alpha-u^{+}<u^{+}$[see Fig. 7(b)], so a reversed rarefaction wave is generated instead of reversed undular bore. The solution for the rarefaction wave is given by formula (81), where $u^{l}=u^{-}$and $u^{r}=u^{*}$. The solibore solution connecting $u^{*}$ and $u^{+}$is the same as in Region 2 . The analytical and numerical plots corresponding to Region 3 are presented in Fig. 10. We note that, since max $u^{+}\left(1-\alpha u^{+}\right)=$ $\frac{1}{4 \alpha}$, the speed of the solibore $s^{k}=\frac{1}{\alpha}+2 u^{+}\left(1-\alpha u^{+}\right)$is always greater than that of the right edge of the rarefaction wave, $s^{+}=s^{r}=6 u^{+}\left(1-\alpha u^{+}\right)$[see (82)]. At the boundary between Regions 3 and 4, when $u^{+}=1 / 2 \alpha$, we have $s^{k}=s^{r}$ and the solibore gets "attached" to the right edge of the rarefaction wave.

Region 4, $1 / 2 \alpha \leqslant u^{+}<u^{-},\left\{u^{-} \leftarrow \mathbf{R W} u^{+}\right\}$. A single reversed rarefaction wave is produced. It is described by the solution (81) with $u^{l}=u^{-}, u^{r}=u^{+}$. The corresponding typical analytical and numerical solution are presented in Fig. 11. Note the small-amplitude dispersive wave train seen to the left of the rarefaction wave in the numerical plot in Fig. 11(b). This wave train is necessary to resolve weak discontinuity at the left edge of the rarefaction wave, while another weak discontinuity at the right edge is smoothed out
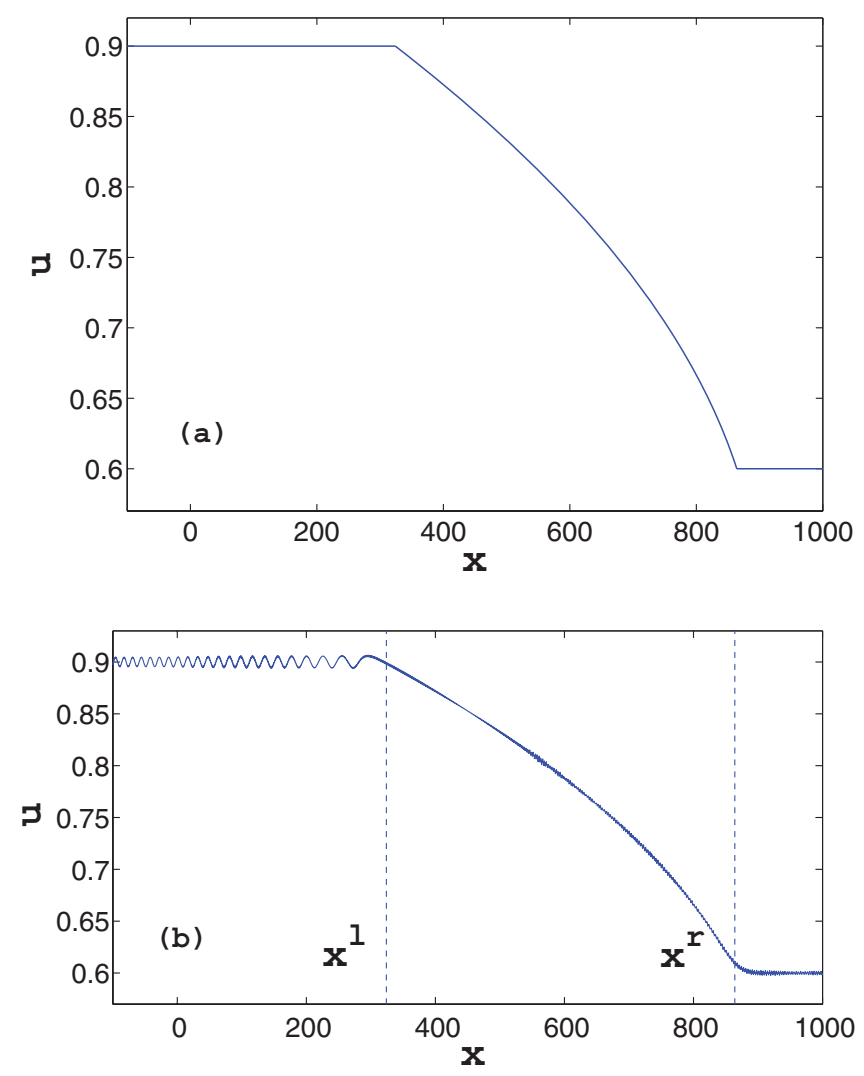

FIG. 11. (Color online) Evolution of an initial discontinuity for the Gardner equation with $\alpha=1$. Region $4:\left\{u^{-} \leftarrow \mathbf{R W} u^{+}\right\}$. The initial step parameters are $u^{-}=0.9, u^{+}=0.6$. (a) Analytical (dispersionless limit) solution. (b) Numerical solution. Dashed lines in (b) correspond to the analytically found locations $x^{l, r}$ of the rarefaction wave edges. Both plots correspond to $t=600$. 
(see [17] for the detailed description of similar effects in the KdV theory).

The wave patterns corresponding to Regions 5-8 represent the "reflections" of the patterns arising in Regions 1-4. More precisely, the counterpart solutions correspond to the "opposite" regions in the parametric map in Fig. 5 and are related to each other by the transformation (5). From this viewpoint, the solutions for Regions 5-8 are not "new." At the same time, the change of the solution polarity (e.g., from the "bright undular bore" to the "dark undular bore") due to the change of initial data is not trivial physically so it deserves separate description. For this reason and for the reader to be able to identify the arising wave patterns directly, without the need to invoke intermediate transformations, we shall proceed with the descriptions of the Regions 5-8 in the same format that was used for Regions 1-4.

Region 5, $1 / 2 \alpha<u^{-}<u^{+}<\frac{1}{\alpha},\left\{u^{-} \leftarrow \mathbf{U B} u^{+}\right\}$. In this region both values $u^{-}$and $u^{+}$are in the domain where the function $w(u)=u(1-\alpha u)$ is monotonically decreasing so a single reversed undular bore is produced. The modulation description of this undular bore is identical to that in Region 2 but to obtain the oscillatory structure one now needs to use the relations (60) between the Riemann invariants and parameters of the periodic solution (13). The amplitude of the lead dark soliton $a^{+}=\left.\left(u_{4}-u_{2}\right)\right|_{m=1}$ is given by the same expression (107). The analytical and numerical solution plots for Region 5 are presented in Fig. 12.
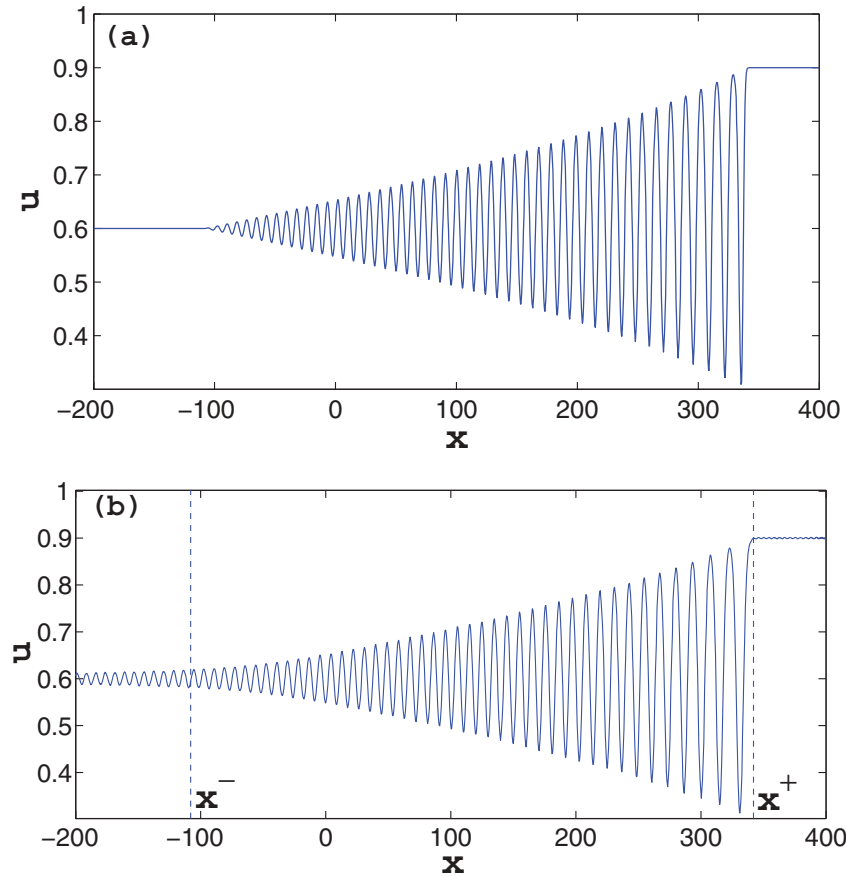

FIG. 12. (Color online) Evolution of an initial discontinuity for the Gardner equation with $\alpha=1$. Region 5: $\left\{u^{-} \leftarrow \mathbf{U B} u^{+}\right\}$. The initial step parameters are $u^{-}=0.6, u^{+}=0.9$. (a) Analytical (modulation theory) solution. (b) Numerical solution of the Gardner equation. Dashed lines in (b) correspond to the analytically found locations $x^{ \pm}$of the undular bore edges. Both plots correspond to $t=300$.
Region 6, $\frac{1}{\alpha}-u^{+}<u^{-}<\frac{1}{2 \alpha}<u^{+},\left\{u^{-} \mathbf{U B} \rightarrow\left(u^{*}\right) \leftarrow\right.$ SB $\left.u^{+}\right\}$. A combination of the normal undular bore and a reversed solibore is produced.

The undular bore connects the state $u^{-}$and an intermediate state $u^{*}=1 / \alpha-u^{+}$and is described by the same set of formulas as a single normal undular bore in Region 1 . The reversed solibore further connects the intermediate state $u^{*}$ with the downstream state $u^{+}$. It is described by formula (20) with "+" sign. The plots of the analytical and numerical solutions are shown in Fig. 13.

Region 7, $u^{-}<\frac{1}{2 \alpha}<u^{+}<1 / \alpha-u^{-},\left\{u^{-} \mathbf{R W} \rightarrow\left(u^{*}\right)\right.$ $\left.\leftarrow \mathbf{S B} u^{+}\right\}$. The resolution pattern is similar to that in Region 6 but, instead of the normal undular bore, a normal rarefaction wave described by formula (80) is generated. The boundary states are $u^{l}=u^{-}, u^{r}=u^{*}=1 / \alpha-u^{+}$. The corresponding analytical and numerical plots are presented in Fig. 14. Note that, similar to Region 3, the solibore always propagates ahead of the rarefaction wave and gets attached to the right edge of the rarefaction wave when $u^{+}=1 / 2 \alpha$.

Region $8, u^{-}<u^{+} \leqslant \frac{1}{2 \alpha}$. A single normal rarefaction wave is produced, $\left\{u^{-} \mathbf{R W} \rightarrow u^{+}\right\}$. It is described by the solution (80) with $u^{l}=u^{-}, u^{r}=u^{+}$. The plots of the analytical and and numerical solutions for Region 8 are shown in Fig. 15.
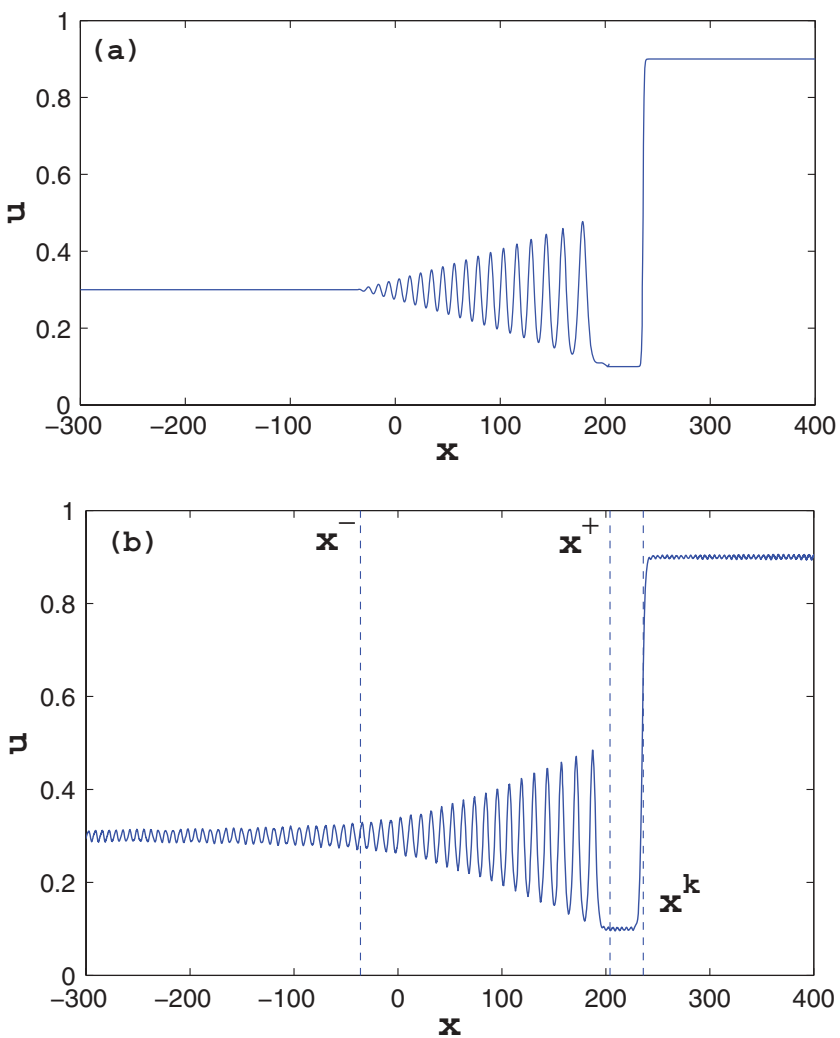

FIG. 13. (Color online) Evolution of an initial discontinuity for the Gardner equation with $\alpha=1$. The initial step parameters are $u^{-}=$ 0.3, $u^{+}=0.9$. Region 6: $\left\{u^{-} \mathbf{U B} \rightarrow\left(u^{*}\right) \leftarrow \mathbf{S B} u^{+}\right\}$. (a) Analytical (modulation theory) solution. (b) Numerical solution of the Gardner equation. Dashed lines in (b) correspond to the analytically found locations of the undular bore edges $x^{ \pm}$and the solibore $x^{k}$. Both plots correspond to $t=200$. 

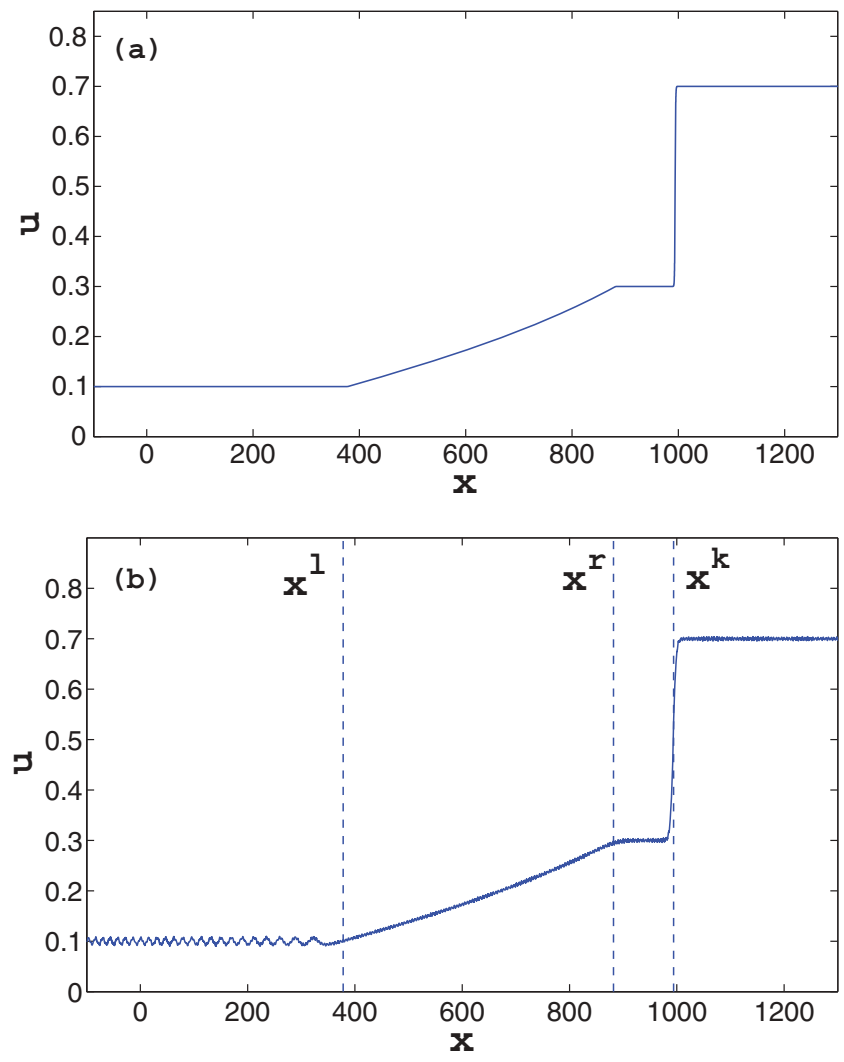

FIG. 14. (Color online) Evolution of an initial discontinuity for the Gardner equation with $\alpha=1$. Region 7: $\left\{u^{-} \mathbf{R W} \rightarrow\left(u^{*}\right) \leftarrow\right.$ SB $\left.u^{+}\right\}$. The initial step parameters are $u^{-}=0.1, u^{+}=0.7$. (a) Analytical (dispersionless limit) solution in the form of a rarefaction wave connected to a solibore. (b) Numerical solution of the Gardner equation. Dashed lines in (b) correspond to the analytically found positions of the solibore $x^{k}$ and rarefaction wave boundaries $x^{l, r}$. Both plots correspond to $t=700$.

\section{Classification for $\alpha<0$}

Now we present the parametric map of solutions of the step problem for the Gardner equation with $\alpha<0$. The most significant change in the structure of solutions compared to the case $\alpha>0$ is that the composite solutions now contain trigonometric undular bores rather than solibores. The plane $\left(u^{+}, u^{-}\right)$of the initial step parameters is again split into eight regions (see Fig. 16). The lines separating different regions are

(a) $u^{-}=u^{+}$separates the regions of pure undular bores and pure rarefaction waves;

(b) $u^{-}=1 / \alpha-u^{+}$corresponds to the steps resolving into single trigonometric bore solutions and separates the regions of composite solutions of different types: undular bore + trigonometric undular bore and rarefaction wave + trigonometric bore; and

(c) $u^{-}=1 / 2 \alpha$ separates regions of pure (undular bore) and composite (undular bore + trigonometric bore) solutions.

Let us now describe in some detail the wave structures corresponding to different regions in Fig. 16.

Region $1, u^{+}<u^{-}<\frac{1}{2 \alpha},\left\{u^{-} \leftarrow \mathbf{R W} u^{+}\right\}$. Both values $u^{-}$and $u^{+}$lie in the domain where the function $w(u)=$ $u(1-\alpha u)$ is monotonically decreasing so there is one-toone correspondence between the dispersionless limits of the
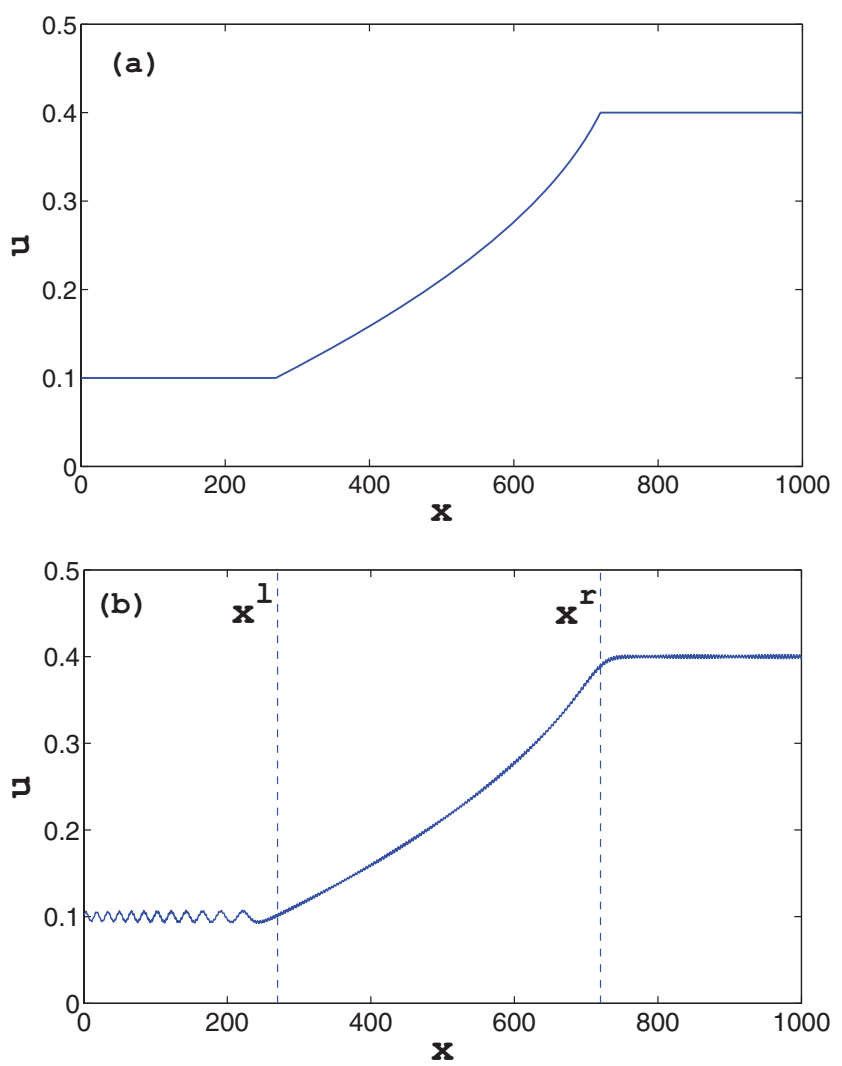

FIG. 15. (Color online) Evolution of an initial discontinuity for the Gardner equation with $\alpha=1$. Region 8: $\left\{u^{-} \mathbf{R W} \rightarrow u^{+}\right\}$. The initial step parameters are $u^{-}=0.1, u^{+}=0.4$. (a) Analytical (dispersionless limit) solution in the form of a rarefaction wave. (b) Numerical solution of the Gardner equation. Dashed lines in (b) correspond to the analytically found positions $x^{l, r}$ of the rarefaction wave boundaries. Both plots correspond to $t=500$.

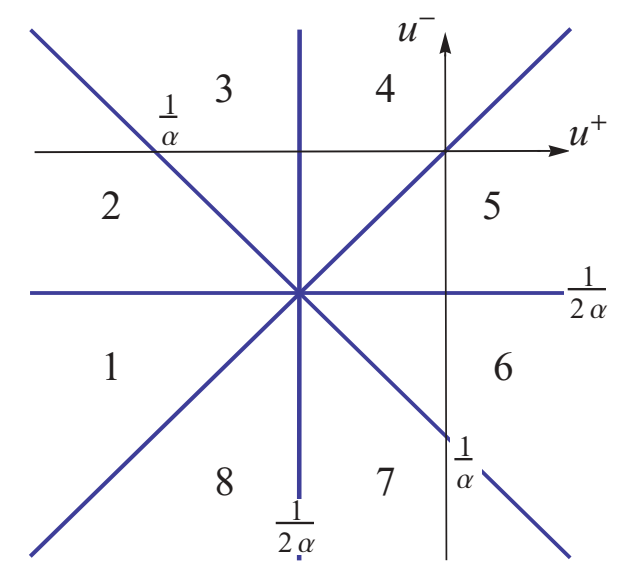

FIG. 16. (Color online) Parametric map of solutions of the step problem for the Gardner equation with $\alpha<0$. The resolution diagrams corresponding to each of the cases on the plane of the initial step parameters $u^{-}$and $u^{+}$are the following: Region 1: $\left\{u^{-} \leftarrow \mathbf{R W} u^{+}\right\}$; Region 2: $\left\{u^{-} \mathbf{T B} \rightarrow\left(u^{*}\right) \leftarrow \mathbf{R W} u^{+}\right\}$; Region 3: $\left\{u^{-}(\mathbf{T B} \mid \mathbf{U B}) \rightarrow u^{+}\right\}$; Region 4: $\left\{u^{-} \mathbf{U B} \rightarrow u^{+}\right\}$; Region 5: $\left\{u^{-}\right.$ RW $\left.\rightarrow u^{+}\right\}$; Region 6: $\left\{u^{-} \leftarrow \mathbf{T B}\left(u^{*}\right) \mathbf{R W} \rightarrow u^{+}\right\}$; Region 7: $\left\{u^{-} \leftarrow\right.$ (TB $\left.\left.\mid \mathbf{U B}\right) u^{+}\right\}$; Region 8: $\left\{u^{-} \leftarrow \mathbf{U B} u^{+}\right\}$. In all relevant cases the intermediate state $u^{*}=1 / \alpha-u^{-}$. 

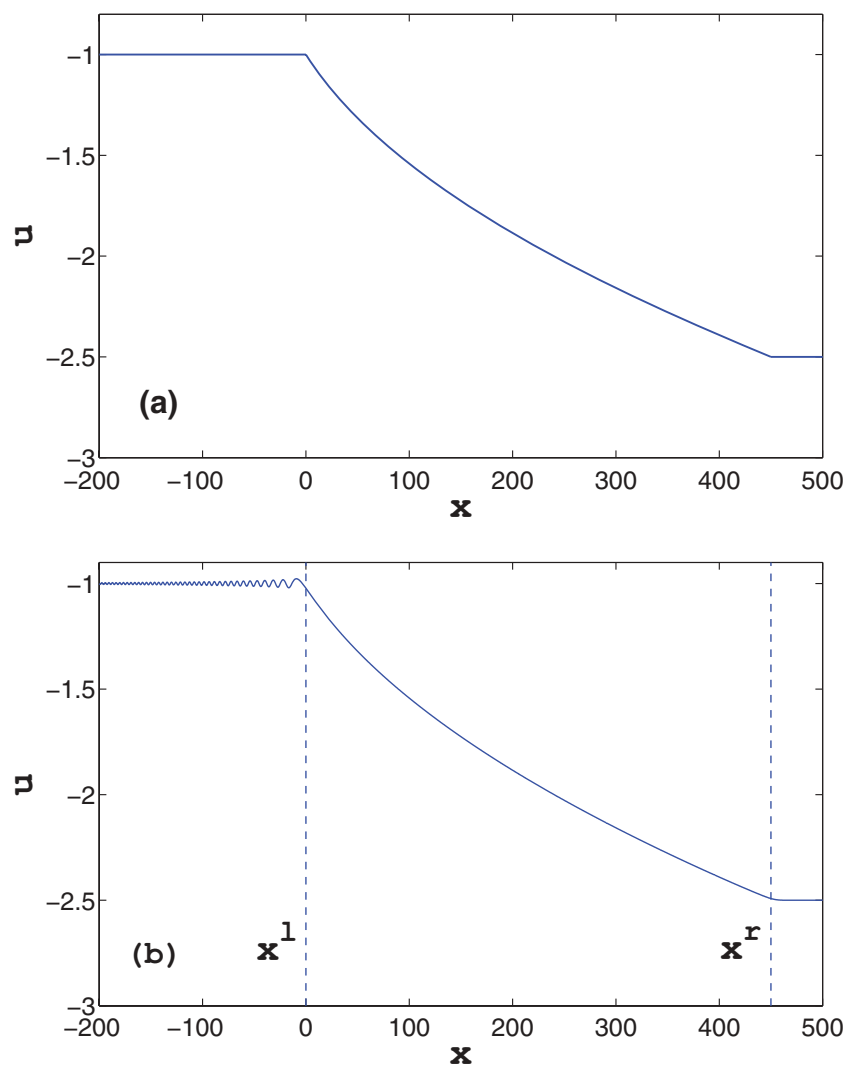

FIG. 17. (Color online) Evolution of an initial discontinuity in the Gardner equation with $\alpha=-1$ : Region 1: $\left\{u^{-} \leftarrow \mathbf{R W} u^{+}\right\}$. The initial step parameters are $u^{-}=-1, u^{+}=-2.5$. (a) Analytical (dispersionless limit) solution. (b) Numerical solution of the Gardner equation. Dashed lines in (b) correspond to the locations of the rarefaction wave edges found analytically. Both plots are made for $t=20$.

Gardner and the KdV equations. This suggests that the Region 1 initial discontinuity is resolved by a single "reversed" simple rarefaction wave. The rarefaction wave is described by the solution (81) with $u^{l}=u^{-}$and $u^{r}=u^{+}$. A typical solution for Region 1 is shown in Fig. 17. The analytically found boundaries of the rarefaction wave are shown by the dashed lines.

Region $2, u^{+}<\frac{1}{2 \alpha}<u^{-}<\frac{1}{\alpha}-u^{+},\left\{u^{-} \mathbf{T B} \rightarrow\left(u^{*}\right) \leftarrow\right.$ $\left.\mathbf{R W} u^{+}\right\}$. Since $u^{+}$and $u^{-}$now lie in different regions of monotonicity of the function $w(u)=u(1-\alpha u)$, the Region 1 solution in the form of a single reversed rarefaction wave is not able to provide the necessary continuous matching between the given states. Instead, one needs to introduce a trigonometric undular bore joining the left constant state $u^{-}$with rarefaction wave at the level $u^{*}=1 / \alpha-u^{-}$(see Fig. 18). The intermediate state $u^{*}$ is found from the condition $w\left(u^{*}\right)=w\left(u^{-}\right)$ [cf. condition $w\left(u^{*}\right)=w\left(u^{+}\right)$for $\alpha>0$ ]. The modulation description of the relevant (normal) trigonometric bore was constructed in Sec. IV A in terms of the Riemann invariants $R_{3} \geqslant R_{2} \geqslant R_{1}$ (83).

The speeds $s^{-}$and $s^{*}$ of the trailing and the leading edges of the trigonometric bore, and the speed of the leading edge of
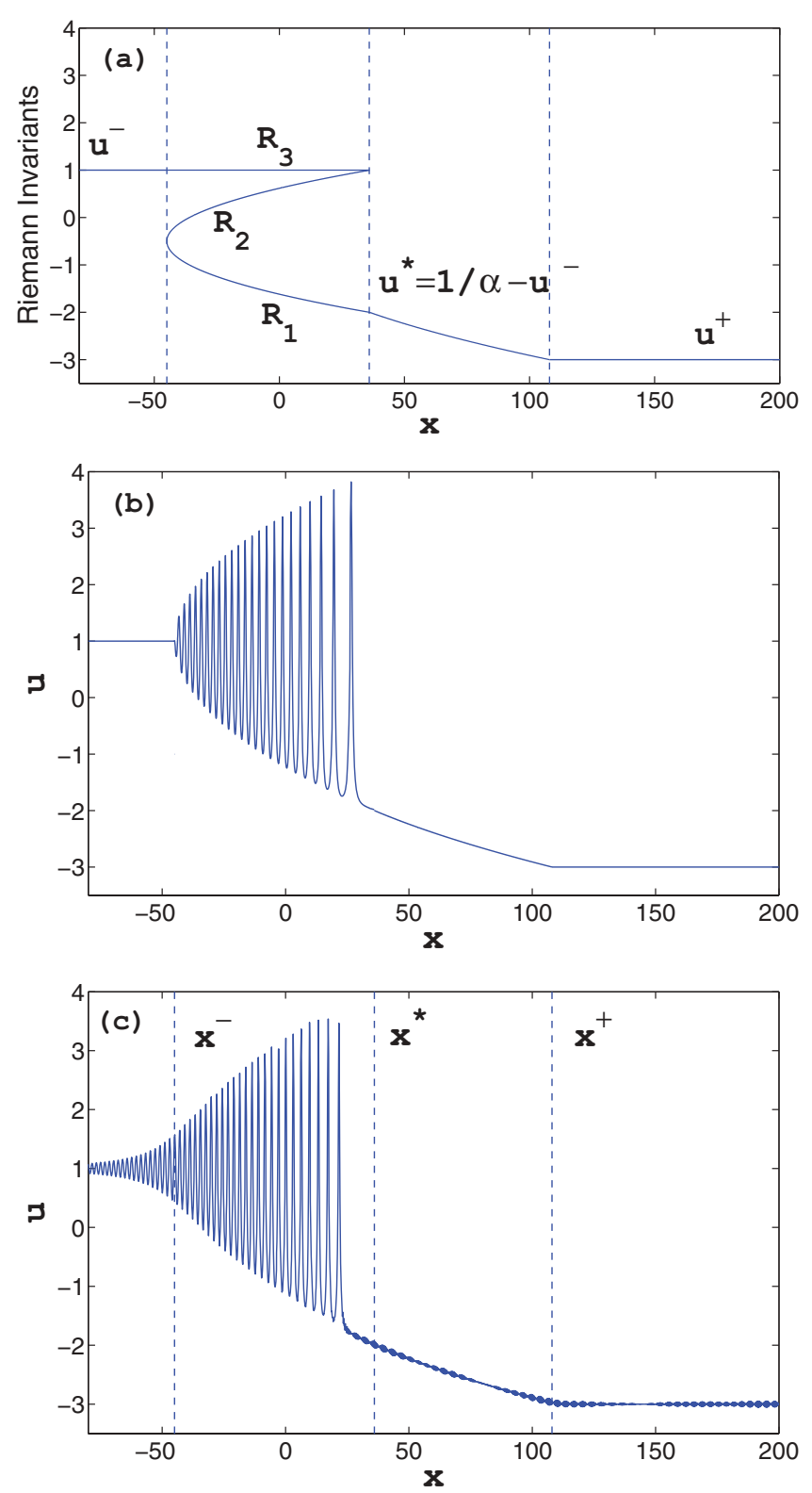

FIG. 18. (Color online) Evolution of an initial discontinuity for the Gardner equation with $\alpha=-1$ for $t=3$. Region $2:\left\{u^{-} \mathbf{T B} \rightarrow\right.$ $\left.\left(u^{*}\right) \leftarrow \mathbf{R W} u^{+}\right\}$. The initial step parameters are $u^{-}=1, u^{+}=-3$. (a) Riemann invariants $R_{1}, R_{2}$, and $R_{3}$. (b) Analytical (modulation theory) solution. (c) Numerical solution of the Gardner equation. Dashed lines on the numerical plot correspond to the analytically found boundaries between different parts of the wave pattern.

the rarefaction wave $s^{+}$are

$$
\begin{aligned}
s^{-} & =\frac{3}{\alpha}-6 u^{-}\left(1-\alpha u^{-}\right), \\
s^{*} & =6 u^{*}\left(1-\alpha u^{*}\right)=6 u^{-}\left(1-\alpha u^{-}\right), \\
s^{+} & =6 u^{+}\left(1-\alpha u^{+}\right) .
\end{aligned}
$$

The analytical and numerical solutions along with the plot for the Riemann invariants $R_{1}, R_{2}, R_{3}$, are shown in Fig. 18.

At $u^{-}=1 / \alpha-u^{+}>u^{+}$the rarefaction wave disappears and one obtains a single normal trigonometric bore as a result 

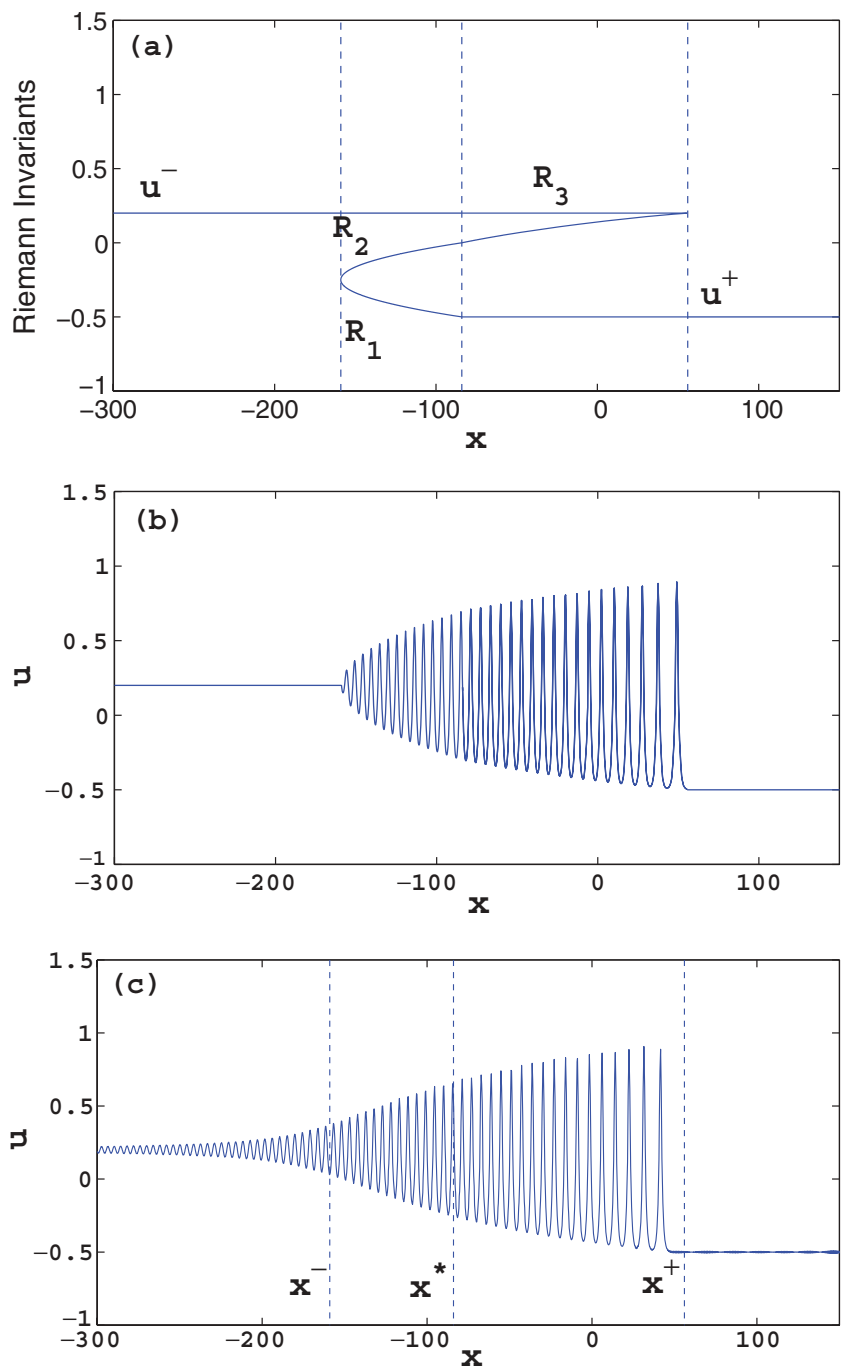

FIG. 19. (Color online) Evolution of an initial discontinuity for the Gardner equation with $\alpha=-2$. Region 3: $\left\{u^{-}(\mathbf{T B} \mid \mathbf{U B}) \rightarrow u^{+}\right\}$. The initial step parameters are $u^{-}=0.2, u^{+}=-0.5$. The plots are shown for $t=50$. (a) Riemann invariants $R_{1}, R_{2}$, and $R_{3}$. (b) Analytical (modulation theory) solution. (c) Numerical solution of the Gardner equation. Dashed lines on the numerical plot correspond to the analytically found boundaries between different parts of the wave pattern.

of the step evolution. The relevant analytical description was presented in Sec. IV A.

Region 3, $\frac{1}{\alpha}-u^{-}<u^{+}<\frac{1}{2 \alpha},\left\{u^{-}(\mathbf{T B} \mid \mathbf{U B}) \rightarrow u^{+}\right\}$. In the Region 3 we get a composite undular bore consisting of normal trigonometric and cnoidal parts matching at the point of the trailing edge $x^{*}=s^{*} t$ of the cnoidal bore. The modulation solution for the entire composite bore is conveniently described in terms of the Riemann invariants $R_{3} \geqslant R_{2} \geqslant R_{1}$ (83). The corresponding analytical and numerical plots are shown in Fig. 19.

The characteristic speeds for this region are

$$
\begin{aligned}
s^{-} & =\frac{3}{\alpha}-6 u^{-}\left(1-\alpha u^{-}\right), \\
s^{*} & =12 u^{+}\left(1-\alpha u^{+}\right)-6 u^{-}\left(1-\alpha u^{-}\right), \\
s^{+} & =2 u^{+}\left(1-\alpha u^{+}\right)+4 u^{-}\left(1-\alpha u^{-}\right) .
\end{aligned}
$$
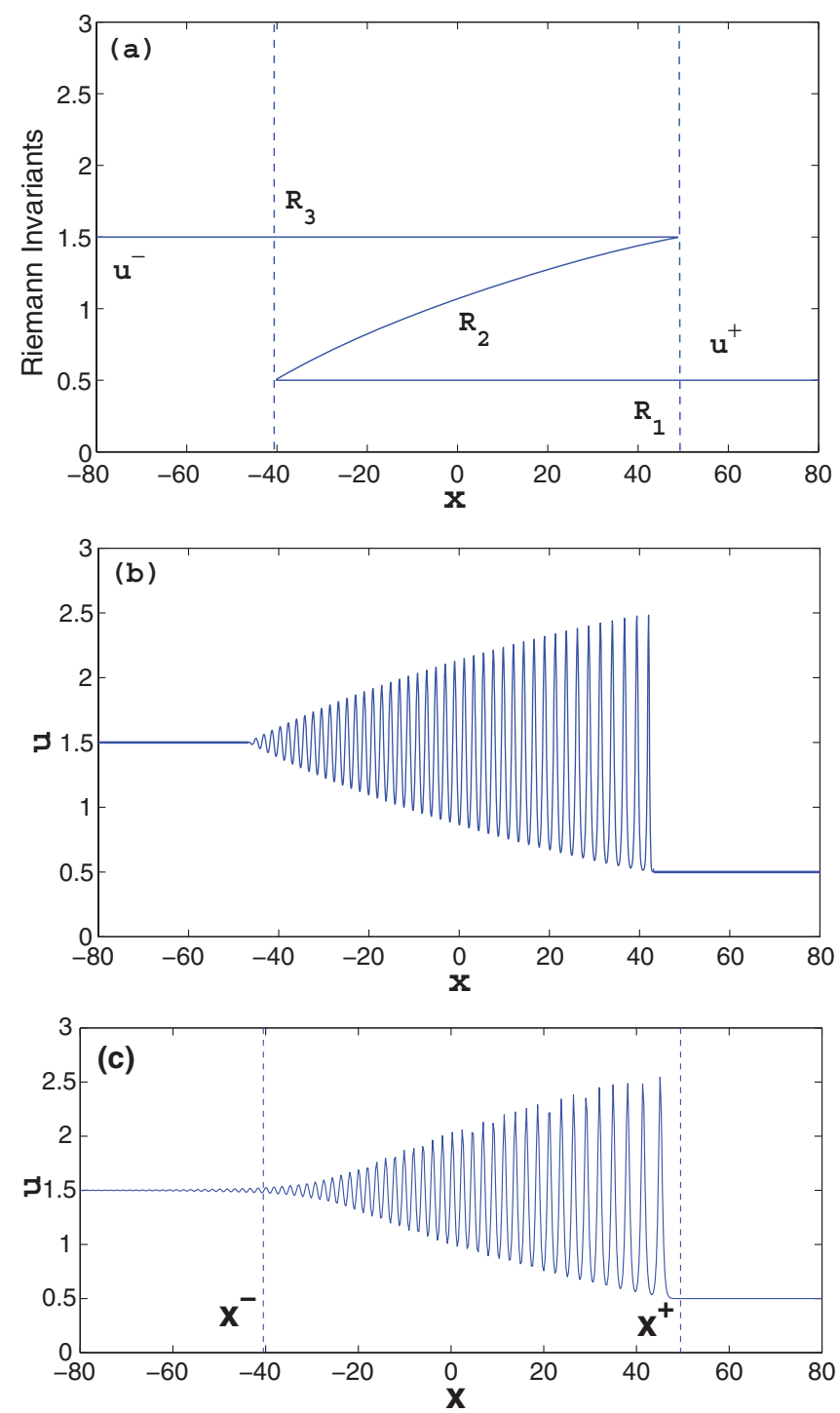

FIG. 20. (Color online) Evolution of an initial discontinuity for the Gardner equation with $\alpha=-1$. Region $4:\left\{u^{-} \mathbf{U B} \rightarrow u^{+}\right\}$. The initial step parameters are $u^{-}=1.5, u^{+}=0.5$. The plots are shown for $t=3$. (a) Riemann invariants $R_{1}, R_{2}$, and $R_{3}$. (b) Analytical (modulation theory) solution. (c) Numerical solution of the Gardner equation. Dashed lines on the numerical plot correspond to the analytically found boundaries between different parts of the wave pattern.

We note that the boundary $x^{*}=s^{*} t$ between the trigonometric bore and the cnoidal bore parts in the composite bore solution can be naturally defined only in the framework of the averaged (Whitham) equations, where it represents a characteristic separating two regions with qualitatively different behavior of the modulation solution [the modulation solution has a weak discontinuity at $x=x^{*}$-see Fig. 19(a)]. Due to the asymptotic nature of the modulation equations (the phase is washed out), this separating line cannot be consistently identified on the level of the genuine (rapidly oscillating) solution of the governing equation. The situation here is similar to that with the definition of the trailing edge of a standard undular bore: the trailing edge cannot be identified with a particular point in the bore but is rather associated 

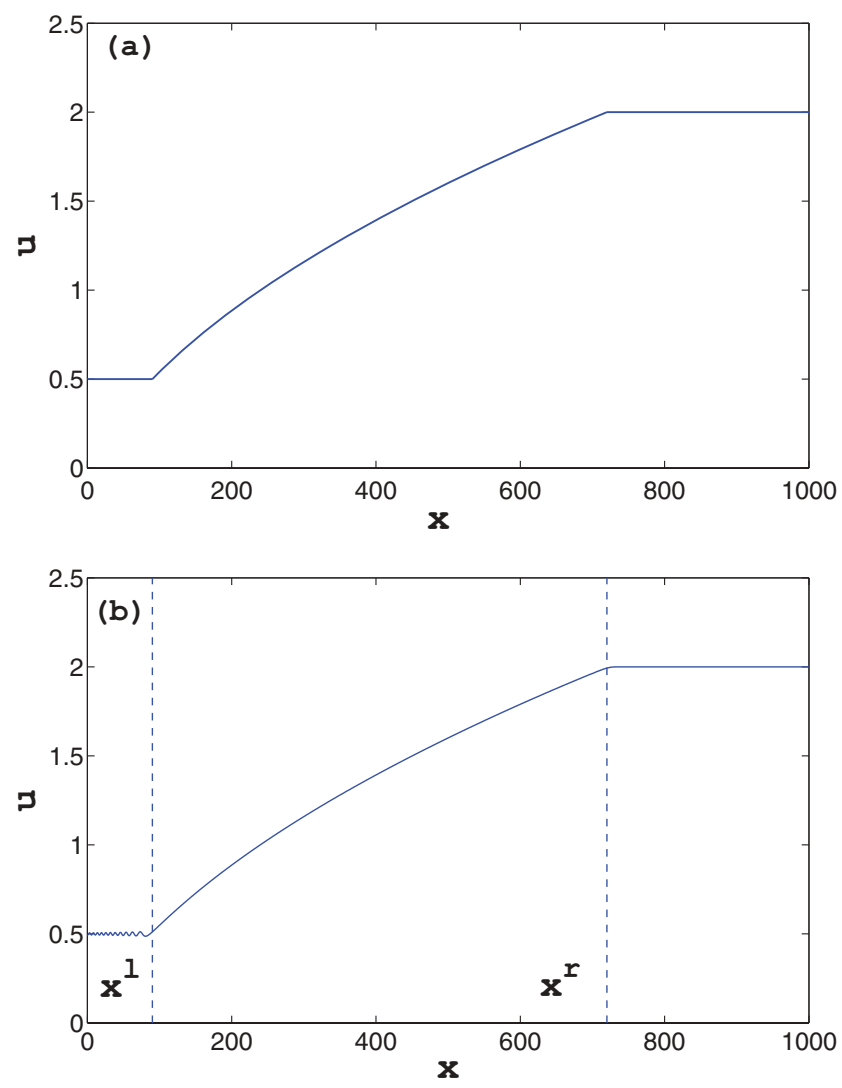

FIG. 21. (Color online) Evolution of an initial discontinuity in the Gardner equation with $\alpha=-1$. Region 5: $\left\{u^{-} \mathbf{R W} \rightarrow u^{+}\right\}$. The initial discontinuity parameters are $u^{-}=0.5, u^{+}=2$. (a) Analytical (dispersionless limit) solution. (b) Numerical solution of the Gardner equation. Dashed lines correspond to the analytically found locations of the rarefaction wave edges. The plots are shown for $t=20$.

with the linear group velocity characteristic of the modulation equations, hence the already mentioned noticeable difference in the behavior of the asymptotic (modulation theory) solution and that of the full numerical solution near the trailing edge [cf. Figs. 19(b) and 19(c)].

Region $4, u^{-}>u^{+}>\frac{1}{2 \alpha},\left\{u^{-} \mathbf{U B} \rightarrow u^{+}\right\}$. Both values $u^{-}>u^{+}$lie in the domain where the function $w(u)=u(1-$ $\alpha u$ ) increases, so the resolution occurs via a single normal cnoidal undular bore (see Fig. 20). To be consistent with other plots in this section we present the Gurevich-Pitaevskii solution (74), (75) for the undular bore in terms of the Riemann invariants $\left\{R_{j}\right\}$ rather than $\left\{r_{j}\right\}$. The one-to-one correspondence between these two sets of the Riemann invariants in Region 4 is given by relations (83).

The edge speeds are [see (77), (78)]

$$
\begin{aligned}
& s^{-}=12 u^{-}\left(1-\alpha u^{-}\right)-6 u^{+}\left(1-\alpha u^{+}\right), \\
& s^{+}=2 u^{-}\left(1-\alpha u^{-}\right)+4 u^{+}\left(1-\alpha u^{+}\right) .
\end{aligned}
$$

Similar to the classification for $\alpha>0$ described in the previous section, the solutions for Regions 5-8 can be obtained by applying the transformation (5) to their counterparts from the opposite regions of the parametric map in Fig. 16. Again, for the convenience of identification we present them below in the same format.

Region 5, $\frac{1}{2 \alpha}<u^{-}<u^{+},\left\{u^{-} \mathbf{R W} \rightarrow u^{+}\right\}$. Both values $u^{-}<u^{+}$lie now in the domain where the function $w(u)=$ $u(1-\alpha u)$ decreases, so the resolution occurs via a single normal rarefaction wave described by (80) with $u^{l}=u^{-}$, $u^{r}=u^{+}$.

The edge speeds are

$$
s^{-}=6 u^{-}\left(1-\alpha u^{-}\right), \quad s^{+}=6 u^{+}\left(1-\alpha u^{+}\right) .
$$
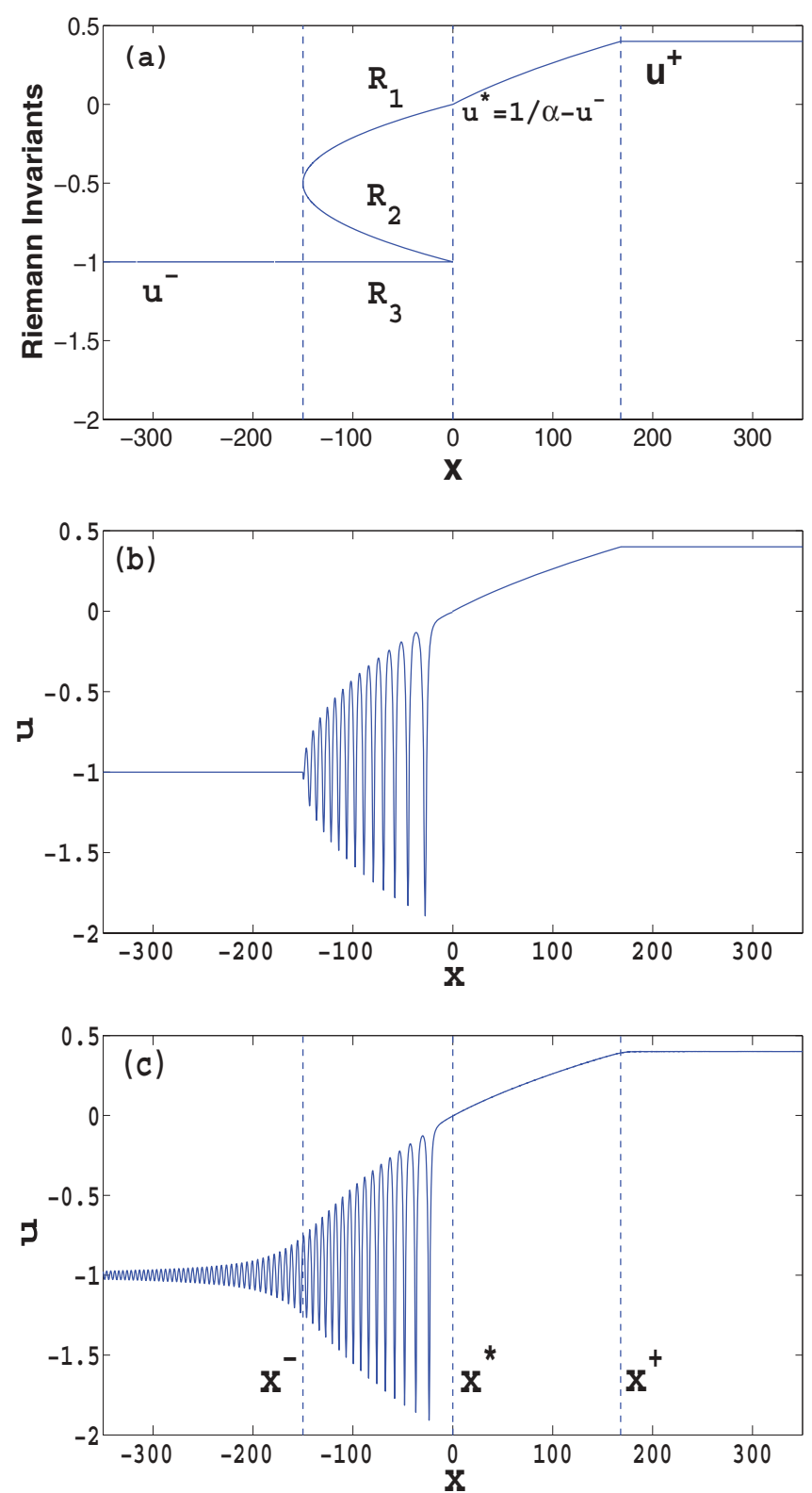

FIG. 22. (Color online) Evolution of an initial discontinuity for the Gardner equation with $\alpha=-1$. The initial step parameters are $u^{-}=-1, u^{+}=0.4$. Region 6: $\left\{u^{-} \leftarrow\right.$ TB $\left.\left(u^{*}\right) \mathbf{R W} \rightarrow u^{+}\right\}$. (a) Riemann invariants $R_{1}, R_{2}$, and $R_{3}$. (b) Analytical (modulation theory) solution. (c) Numerical solution of the Gardner equation. $t=50$. Dashed lines on the numerical plot correspond to the analytically found boundaries between different parts of the wave pattern. 

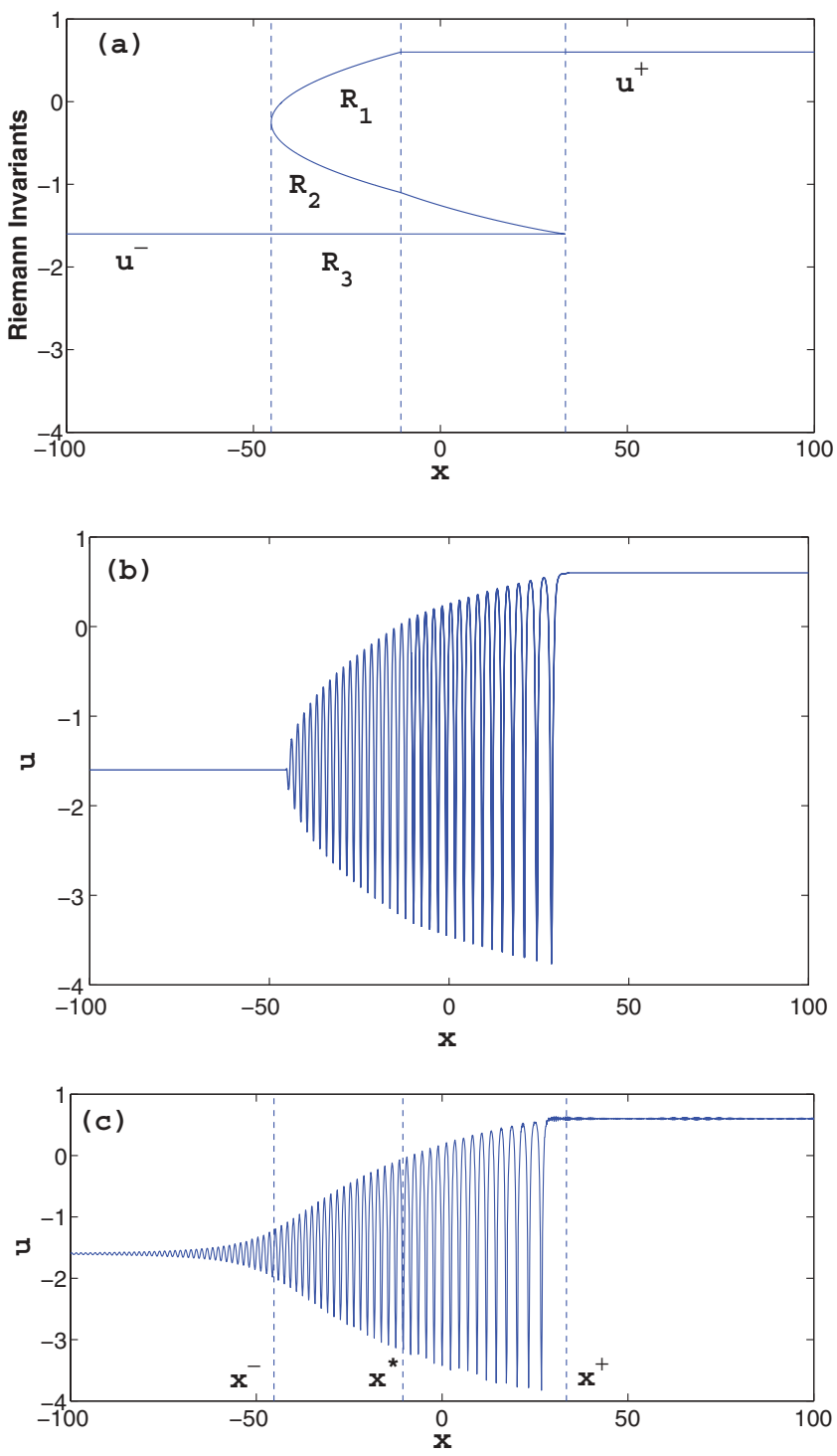

FIG. 23. (Color online) Evolution of an initial discontinuity for the Gardner equation with $\alpha=-2$. Region 7: $\left\{u^{-} \leftarrow\right.$ (TB $\left.\left.\mid \mathbf{U B}\right) u^{+}\right\}$. The initial step parameters are $u^{-}=-1.6, u^{+}=0.6$. The plots are shown for $t=2$. (a) Riemann invariants $R_{1}, R_{2}$, and $R_{3}$. (b) Analytical (modulation theory) solution. (c) Numerical solution of the Gardner equation. Dashed lines on the numerical plot correspond to the analytically found boundaries between different parts of the wave pattern.

The corresponding numerical solution is shown in Fig. 21 along with the boundaries of the analytical RW solution marked by dashed lines.

Region $\quad 6, \quad \frac{1}{\alpha}-u^{+}<u^{-}<\frac{1}{2 \alpha}<u^{+}, \quad\left\{u^{-} \leftarrow\right.$ $\left.\mathbf{T B}\left(u^{*}\right) \mathbf{R W} \rightarrow u^{+}\right\}$. The resolution pattern corresponding to this region is similar to that in Region 2 but now the resolution occurs via the combination of the reversed trigonometric bore and normal rarefaction wave joined at the level $u^{*}=1 / \alpha-u^{-}$. The modulation description of such a bore is constructed in Sec. IV A in terms of the Riemann invariants $R_{1} \geqslant R_{2} \geqslant R_{3}$ (84). The relevant plots are shown in Fig. 22.
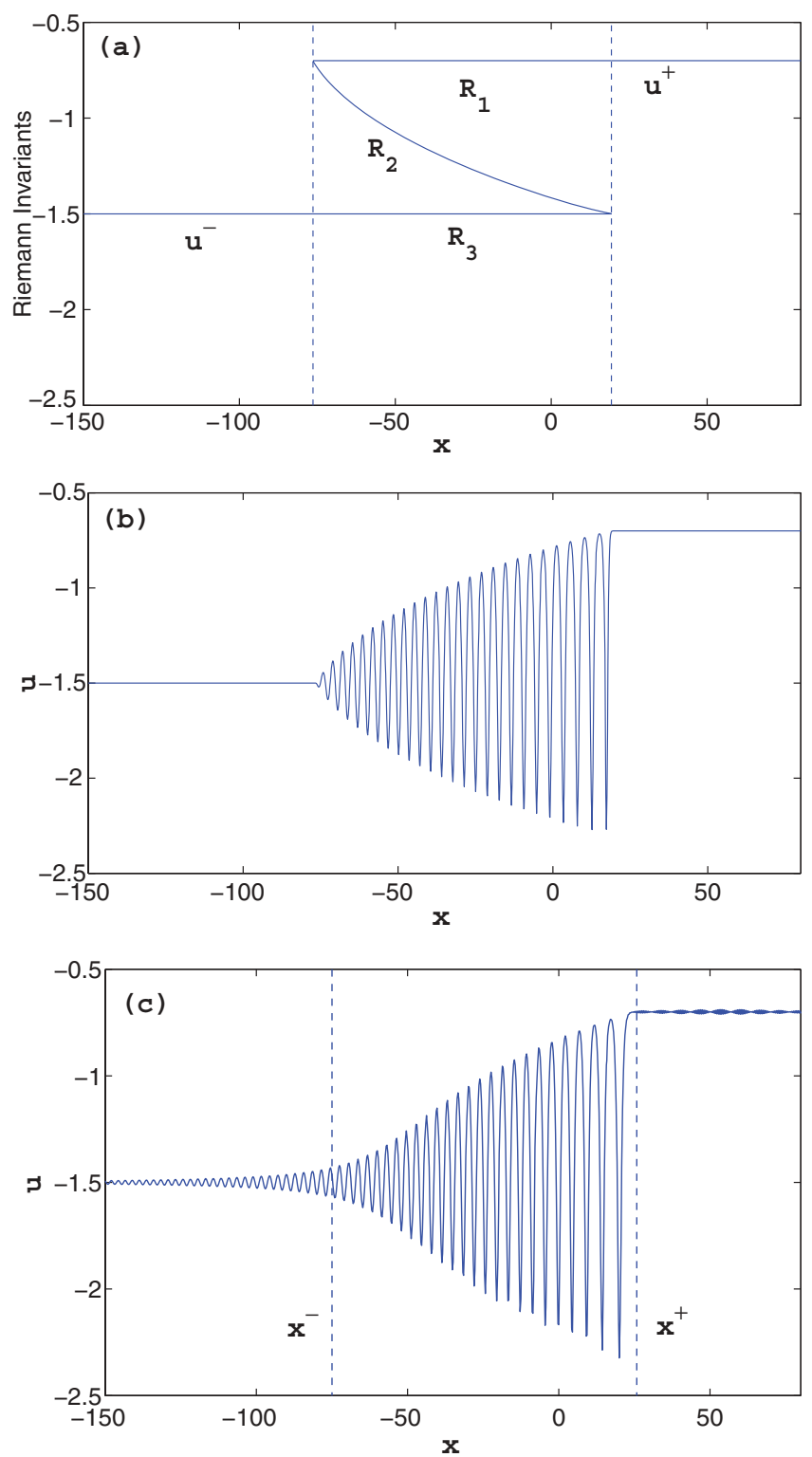

FIG. 24. (Color online) Evolution of an initial discontinuity for the Gardner equation with $\alpha=-1$. Region 8: $\left\{u^{-} \leftarrow \mathbf{U B} u^{+}\right\}$. The initial step parameters are $u^{-}=-1.5, u^{+}=-0.7$. The plots are shown for $t=10$. (a) Riemann invariants $R_{1}, R_{2}$, and $R_{3}$. (b) Analytical (modulation theory) solution. (c) Numerical solution of the Gardner equation. Dashed lines on the numerical plot correspond to the analytically found boundaries between different parts of the wave pattern.

The edge speeds are equal to

$$
\begin{aligned}
s^{-} & =\frac{3}{\alpha}-6 u^{-}\left(1-\alpha u^{-}\right), \\
s^{*} & =6 u^{-}\left(1-\alpha u^{-}\right), \\
s^{+} & =6 u^{+}\left(1-\alpha u^{+}\right) .
\end{aligned}
$$

The part of the line $u^{-}=1 / \alpha-u^{+}$, where $u^{-}<u^{+}$, separating Regions 6 and 7 corresponds to a pure reversed trigonometric bore described in Sec. IV A. 
Region $7, u^{-}<\frac{1}{2 \alpha}<u^{+}<\frac{1}{\alpha}-u^{-},\left\{u^{-} \leftarrow(\mathbf{T B} \mid \mathbf{U B}) u^{+}\right\}$. Region 7 corresponds to the formation of a composite reversed trigonometric-undular bore (cf. Region 3 for the counterpart normal resolution pattern). The corresponding plots are shown in Fig. 23. The edge speeds are

$$
\begin{aligned}
s^{-} & =\frac{3}{\alpha}-6 u^{+}\left(1-\alpha u^{+}\right), \\
s^{*} & =12 u^{+}\left(1-\alpha u^{+}\right)-6 u^{-}\left(1-\alpha u^{-}\right), \\
s^{+} & =2 u^{+}\left(1-\alpha u^{+}\right)+4 u^{-}\left(1-\alpha u^{-}\right) .
\end{aligned}
$$

Region $8, u^{-}<\frac{1}{\alpha}-u^{+}, \quad u^{+}<\frac{1}{2 \alpha} ;\left\{u^{-} \leftarrow \mathbf{U B} u^{+}\right\}$. This region corresponds to the formation of a reversed cnoidal bore (cf. Region 4); the plots are presented in Fig. 24. Note that the Riemann invariants $R_{1} \geqslant R_{2} \geqslant R_{3}$ (84) were used in the construction of the modulation solution for the reversed bore; as a matter of fact, it is equivalent to the Gurevich-Pitaevskii solution (74), (75) in the original variables $r_{3}>r_{2}>r_{1}$ (see Fig. 3).

The edge speeds are

$$
\begin{aligned}
& s^{-}=12 u^{+}\left(1-\alpha u^{+}\right)-6 u^{-}\left(1-\alpha u^{-}\right), \\
& s^{+}=2 u^{+}\left(1-\alpha u^{+}\right)+4 u^{-}\left(1-\alpha u^{-}\right) .
\end{aligned}
$$

\section{CONCLUSIONS AND OUTLOOK}

We have constructed a full analytical description of the step problem for the Gardner equation (1) for both signs of the coefficient $\alpha$ before the cubic term. The complete classification of arising solutions for different parameters $u^{+}, u^{-}$defining the initial step (6) includes 16 possible cases (eight for each sign of $\alpha$ ). Each sector on the $\left(u^{+}, u^{-}\right)$plane of the parametric map of solutions corresponds to a unique wave pattern representing one of the following: undular bore, a rarefaction wave, a solibore, a trigonometric bore, or a combination of two of the above wave structures. The wave pattern arising in each case depends on the position of the initial step parameters $u^{-}, u^{+}$relative to each other and to the turning point $1 /(2 \alpha)$ of the function $6 u(1-\alpha u)$ defining the characteristic speed of the dispersionless limit of the Gardner equation. The analytical description of undular bores is made using the Whitham modulation theory. The observed rich phenomenology of solutions arising in the step problem for the Gardner equation is due to the fact that the modulation Whitham system associated with the Gardner equation, unlike that for the $\mathrm{KdV}$ equation, is neither strictly hyperbolic nor genuinely nonlinear. Our analytical solutions are supported by numerical simulations.

One of the important applications of the obtained solutions is an analytical description of transcritical flow in a stratified fluid in the framework of the forced Gardner equation (cf. [29,30]). Other possible applications include the consideration of the interaction of internal undular bores with variable topographies (cf. [41]) and the description of the perturbed modulation regimes for internal waves (e.g., due to the inclusion of weak dissipation). In the latter case, the description will require a perturbed modulation theory approach developed in [42]. The obtained classification will also provide guidance for the similar classifications for fully nonlinear non-integrable counterparts of the Gardner equation (such as the Myatta-Choi-Camassa system [36]) to which the analytic technique of the undular bore description developed in [20] is applicable (see [22]).

\section{ACKNOWLEDGMENTS}

A.M.K. thanks National Taiwan University and Taida Institute for Mathematical Sciences, where this work was started, for kind hospitality. T.L.H. acknowledges support of the National Science Council of Taiwan under Grant No. NSC-100-2632-E-035-001-MY3. S.C.G. acknowledges support from the National Center for Theoretical Science, Taiwan.
[1] R. M. Miura, C. S. Gardner, and M. D. Kruskal, J. Math. Phys. 9, 1204 (1968).

[2] S. Watanabe, J. Phys. Soc. Jpn. 53, 950 (1984).

[3] M. S. Ruderman, T. Talipova, and E. Pelinovsky, J. Plasma Phys. 74, 639 (2008).

[4] R. Grimshaw, in Environmental Stratified Flows, edited by R. Grimshaw (Kluwer, Dordrecht, 2002), p. 1.

[5] E. Demler and A. Maltsev, Ann. Phys. (NY) 326, 1775 (2011).

[6] K. R. Helfrich and W. K. Melville, Annu. Rev. Fluid Mech. 38, 395 (2006).

[7] J. R. Apel, L. A. Ostrovsky, Y. A. Stepanyants, and J. F. Lynch, J. Acoust. Soc. Am. 121, 695 (2007).

[8] T. Kakutani and N. Yamasaki, J. Phys. Soc. Jpn. 45, 674 (1978).

[9] P. E. Holloway, E. Pelinovsky, and T. Talipova, J. Geophys. Res. 104, 18333 (1999).

[10] N. F. Smyth and P. E. Holloway, J. Phys. Oceanogr. 18, 947 (1988).

[11] G. A. El, R. H. J. Grimshaw, and N. Smyth, Phys. Fluids 18, 027104 (2006).
[12] R. H. J. Grimshaw and N. F. Smyth, J. Fluid Mech. 169, 429 (1986).

[13] P. G. Baines, Topographic Effects in Stratified Flows (Cambridge University Press, Cambridge, 1995).

[14] M. Hoefer and M. Ablowitz, Scholarpedia J. 4, 5562 (2009).

[15] G. B. Whitham, Proc. R. Soc. London, Ser. A 283, 238 (1965).

[16] G. B. Whitham, Linear and Nonlinear Waves (WileyInterscience, New York, 1974).

[17] A. V. Gurevich and L. P. Pitaevskii, Zh. Eksp. Teor. Fiz. 65, 590 (1973) [Sov. Phys. JETP 38, 291 (1974)].

[18] A. M. Kamchatnov, Nonlinear Periodic Waves and Their Modulations-An Introductory Course (World Scientific, Singapore, 2000).

[19] G. A. El, R. H. G. Grimshaw, and M. V. Pavlov, Stud. Appl. Math. 106, 157 (2001).

[20] G. A. El, Chaos 15, 037103 (2005).

[21] G. A. El, A. Gammal, E. G. Khamis, R. A. Kraenkel, and A. M. Kamchatnov, Phys. Rev. A 76, 053813 (2007). 
[22] J. G. Esler and J. D. Pierce, J. Fluid Mech. 667, 555 (2011).

[23] C. F. Driscoll and T. M. O’Neil, J. Math. Phys. 17, 1196 (1975).

[24] A. M. Kamchatnov, A. Spire, and V. V. Konotop, J. Phys. A 37, 5547 (2004).

[25] T. R. Marchant, Wave Motion 45, 540 (2008).

[26] Y. Kodama, V. U. Pierce, and F.-R. Tian, SIAM J. Math. Anal. 40, 1750 (2008).

[27] M. V. Pavlov, Doklady Akad. Nauk 339, 157 (1994) [Russian Acad. Sci. Dokl. Math. 50, 400 (1995)].

[28] T. R. Marchant and N. F. Smyth, J. Fluid Mech. 221, 263 (1990).

[29] W. K. Melville and K. Helfrich, J. Fluid Mech. 178, 31 (1987).

[30] R. H. J. Grimshaw, K. H. Chan, and K. W. Chow, Phys. Fluids 14, 755 (2002).

[31] V. M. Vassilev, P. A. Djondjorov, M. Ts. Hadzhilazova, and I. M. Mladenov, AIP Conf. Proc. No. 1404 (AIP, Melville, NY, 2011), p. 86.

[32] V. E. Zakharov, S. V. Manakov, S. P. Novikov, and L. P. Pitaevskii, Theory of Solitons (Nauka, Moscow, 1980).
[33] H. Flaschka, M. G. Forest, and D. W. McLaughlin, Commun. Pure Appl. Math. 33, 739 (1980).

[34] P. A. Clarkson, N. Joshi, and M. Mazzocco, Séminaires et Congrès 14, 53 (2006).

[35] A. M. Kamchatnov and R. A. Kraenkel, J. Phys. A 35, L13 (2002).

[36] W. Choi and R. Camassa, J. Fluid Mech. 396, 1 (1999).

[37] N. M. Ercolani, S. Jin, C. D. Levermore, and W. D. MacEvoy, Int. Math. Res. Notices 47, 2529 (2003).

[38] W. E. Schiesser, The Numerical Method of Lines: Integration of Partial Differential Equations (Academic, New York, 1991).

[39] B. Fornberg and G. B. Whitham, Philos. Trans. R. Soc. London, Ser. A 289, 373 (1978).

[40] T. Grava and C. Klein, Commun. Pure Appl. Math. 60, 1623 (2007).

[41] G. A. El, R. H. J. Grimshaw, and W. K. Tiong, J. Fluid Mech., doi: 10.1017/jfm.2012.338.

[42] A. M. Kamchatnov, Physica D 188, 247 (2004). 